\title{
IRNSS Stand-alone Positioning: First results in Australia
}

\author{
S. Zaminpardaz $\cdot$ P.J.G. Teunissen $\cdot$ N. Nadarajah
}

10 Apr 2016

\begin{abstract}
The Indian Regional Navigation Satellite System (IRNSS) currently under development is expected to reach full operational capability before 2017. As a large part of the Australian continent lies in IRNSS' service area, it is important to gain an understanding of its navigational potential and actual positioning capabilities for Australian users. The goals of this contribution are therefore to provide insight into IRNSS, to demonstrate its current positioning performance using actual L5 pseudorange tracking data, and to analyse its expected positioning performance for when the system is fully operational. As such this contribution provides the very first results of the IRNSS stand-alone positioning capabilities over Australia.
\end{abstract}

Keywords: Indian Regional Navigation Satellite System (IRNSS Position Dilution of Precision (PDOP), Single Point Positioning (SPP), Relative Point Positioning (RPP), L5 Pseudorange, Phase-smoothed pseudorange

\section{Introduction}

The Indian Regional Navigation Satellite System (IRNSS) is a satellite-based navigation system currently under devel-

\section{S. Zaminpardaz}

GNSS Research Centre, Department of Spatial Sciences, Curtin University of Technology, Perth, Australia

E-mail: safoora.zaminpardaz@curtin.edu.au

P. J. G. Teunissen

GNSS Research Centre, Department of Spatial Sciences, Curtin University of Technology, Perth, Australia; Department of Geoscience and Remote Sensing, Delft University of Technology, Delft, The Netherlands

N. Nadarajah

GNSS Research Centre, Department of Spatial Sciences, Curtin University of Technology, Perth, Australia opment by the Indian Space Research Organisation (ISRO). IRNSS will consist of three geosynchronous orbit (GEO) satellites and four inclined geosynchronous orbit (IGSO) satellites (see Figure 1(a),(b)), providing two types of services: a standard positioning service (SPS) for civilian users and a restricted service (RS) for authorised users (ISRO, 2014a). The expected positioning accuracy of IRNSS is $10 \mathrm{~m}(2 \sigma)$ over the Indian landmass and $20 \mathrm{~m}$ over the Indian Ocean, covering regions located up to $1500 \mathrm{~km}$ from India between longitude $30^{\circ} \mathrm{E}$ and $130^{\circ} \mathrm{E}$ and between latitude $30^{\circ} \mathrm{S}$ and $50^{\circ} \mathrm{N}$ (Sarma et al, 2010; Ganeshan, 2012; Saikiran and Vikram, 2013).

A few published studies have hitherto been devoted to the IRNSS. Next to the simulation-based studies (Mozo Garcia et al, 2010; Sarma et al, 2010; Sekar et al, 2012; Rethika et al, 2013; Rao, 2013), some newer studies make use of real IRNSS data. Thoelert et al (2014) characterised the IRNSS signals by means of data from a high-gain antenna, and assesses the clock stability of IRNSS-1A. In (Kumari et al, 2015), a precise model for solar radiation pressure was proposed, of which the accuracy was then tested using the IRNSS$1 \mathrm{~A}$ and $1 \mathrm{~B}$ observations.

Babu et al (2015) compared orbit determination methods for IRNSS-1A, 1B and 1C. In order to validate the orbit accuracy with modernised ephemeris parameters, Chandrasekhar et al (2015) made use of the IRNSS-1A, 1B and $1 \mathrm{C}$ real measurements. Montenbruck et al (2015) used the observations of the IRNSS-1A and 1B to investigate the quality of the IRNSS navigation messages and Nadarajah et al (2015), after assessing the IRNSS noise characteristics, combined the L5/E5 signals of IRNSS, GPS, Galileo and QZSS for instantaneous attitude determination.

As IRNSS will soon become fully operational (before the end of 2016), it is important to gain an understanding of its navigational potential and actual positioning capabilities. This is not only of general interest, but also specifically for 
(a)

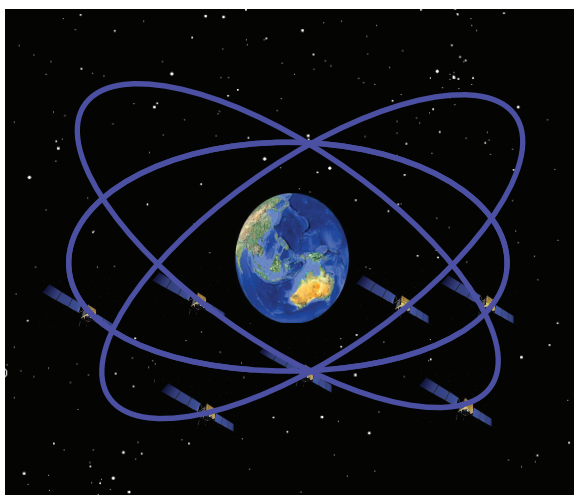

(b)

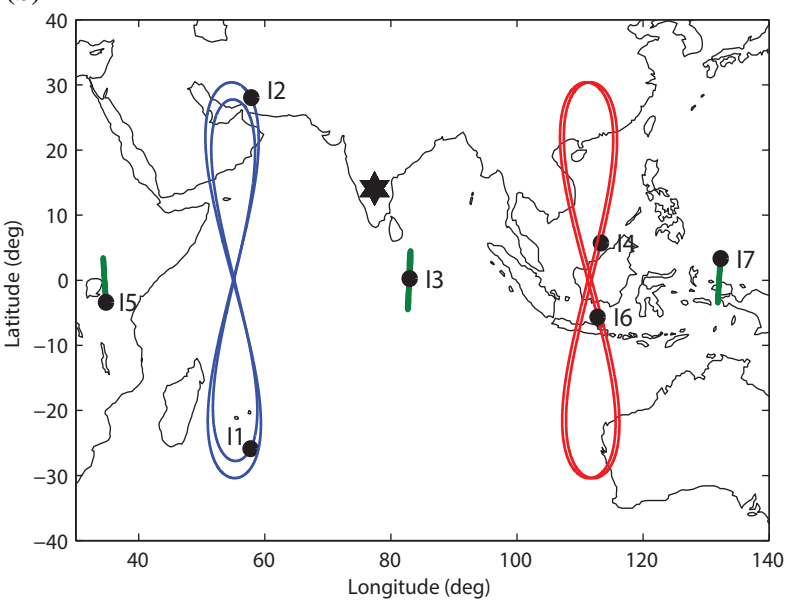

Fig. 1 (a) The 7 satellite IRNSS constellation when fully operational. (b) The three GEO and four IGSO satellite ground tracks of fully operational IRNSS.

Australian users since a large part of the Australian continent lies in IRNSS's service area. The goals of this contribution are therefore to provide insight into IRNSS, to demonstrate its current positioning performance using actual L5 tracking data, and to analyse its expected positioning performance for when the system is fully operational. As such this contribution provides the very first results of the IRNSS stand-alone positioning capabilities over Australia.

This contribution is organised as follows. The IRNSS system is briefly described in section 2. In section 3 we formulate the single point positioning (SPP) model of observation equations and analyse the observability of the current IRNSS constellation for several Australian locations in its secondary service area. This analysis includes the identification and explanation of occurring periods of poor receiversatellite geometries. In section 4 we analyse, formally as well as empirically, the SPP precision that can be achieved with the current constellation. In an analogous fashion, section 5 presents a formal and empirical analysis of the relative point positioning (RPP) precision that is achievable with the current constellation. Here we also show by how much the results can be further improved when use is made of carrier-phase smoothing on the observed L5 pseudoranges. The expected performance of the fully operational system is presented in section 6, first through a PDOP analysis and then by means of a positioning precision analysis with and without carrier-phase smoothing. Finally, the main results are summarised in section 7 .

\section{The IRNSS system}

The Indian Regional Navigation Satellite System (IRNSS) is a satellite-based navigation system being developed by India. IRNSS is planned to consist of three geosynchronous orbit (GEO) satellites and four inclined geosynchronous orbit (IGSO) satellites, providing two services: a standard positioning service (SPS) for civilian users and a restricted service (RS) for authorised users (ISRO, 2014a).

The IRNSS satellites transmit navigation signals, based on code division multiple access (CDMA), on L5 (1176.45 $\mathrm{MHz}$ ) and on $\mathrm{S}(2492.028 \mathrm{MHz})$, with a binary phase-shift key (BPSK (1)) modulation for SPS users, and with a binary offset carrier (BOC $(5,2)$ ) modulation for RS users (ISRO, 2014a). The orbital period of the IRNSS satellites is one sidereal day ( $23 \mathrm{~h}$ and $56 \mathrm{~min}$ ), such that the IRNSS satellite ground tracks repeat every solar day $(24 \mathrm{~h})$ four minutes earlier.

Figure 1(a) and (b) show the fully operational seven satellite IRNSS constellation together with the corresponding ground tracks, respectively. Three satellites will be placed as GEOs located at longitudes $32.5^{\circ} \mathrm{E}, 83^{\circ} \mathrm{E}$ and $131.5^{\circ} \mathrm{E}$, respectively, while the remaining four satellites will be IGSOs, in pairs, placed at an inclination angle of $29^{\circ}$ w.r.t the equator, with their longitude crossings at $55^{\circ} \mathrm{E}$ and $111.75^{\circ} \mathrm{E}$ respectively (ISRO, 2014a). The complete system is planned to be fully operational by the end of 2016 (GPS World, 2015).

The current operational IRNSS constellation (November 2015) comprises of three IGSO satellites, IRNSS-1A/1B/1D (I1/I2/I4), and one GEO satellite, IRNSS-1C (I3). The satellites I1, I2, I3, and I4 were launched by the launchers PSLVC22, PSLV-C24, PSLV-C26, and PSLV-C27 on 1 July 2013, 4 April 2014, 16 October 2014, and 28 March 2015, respectively (ISRO, 2014b,c,d, 2015). The launches for the remaining three satellites are planned in the following periods: IRNSS-1E (January 2016), IRNSS-1F (February-March 2016) and IRNSS-G (March-April 2016). Figure 2(a) and (b) show satellite I1 and the launch vehicle PSLV-C22, respectively (ISRO, 2014b). 
(a)

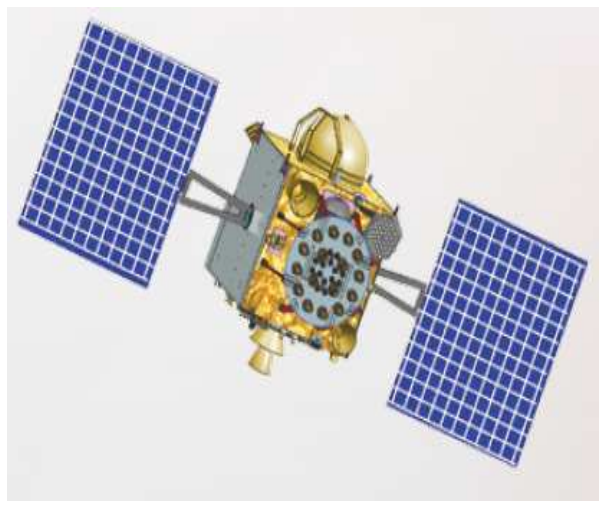

(b)

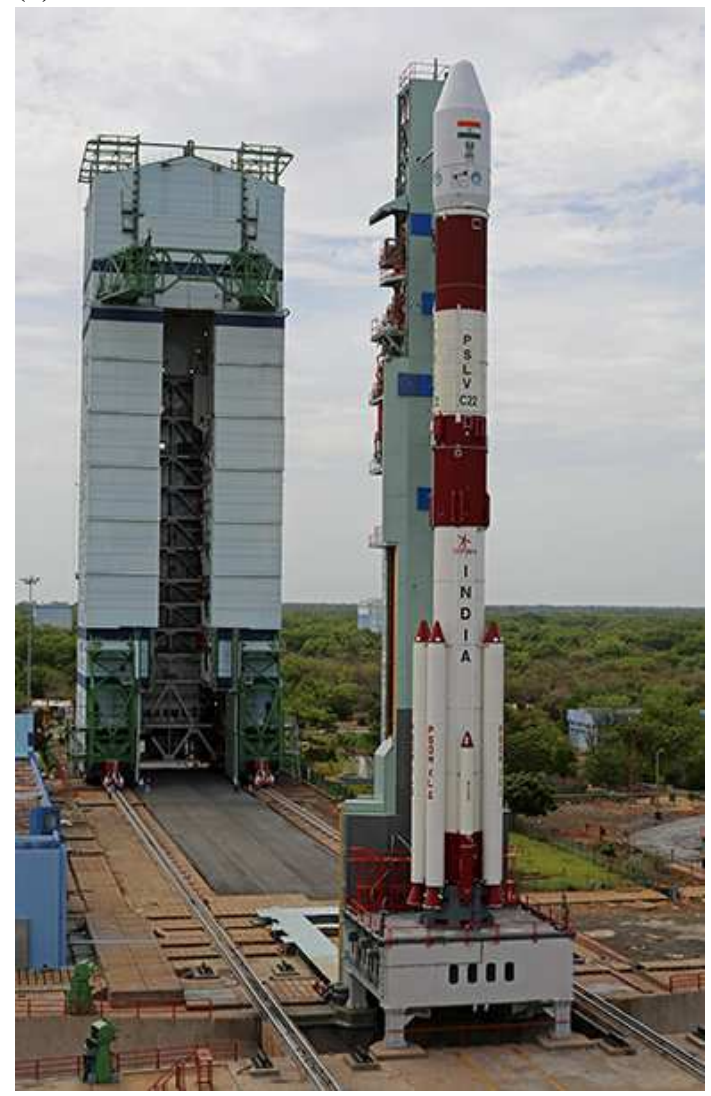

Fig. 2 (a) Artistic view of satellite I1. (b) The PSLV-C22 rocket used to launch I1 (images credit: ISRO).

The IRNSS is established with the objective of offering positioning, navigation and timing (PNT) services to the users in its service area. The IRNSS classifies its service areas broadly into the two regions shown in Figure 3. The primary service area of IRNSS encompasses the Indian landmass and a region lying within a distance of $1500 \mathrm{~km}$ from its geo-political boundary. In this area the system is designed to provide its users with a position accuracy of less than $10 \mathrm{~m}(2 \sigma)$. The secondary service area extends between latitudes $30^{\circ} \mathrm{S}$ to $50^{\circ} \mathrm{N}$ and longitudes $30^{\circ} \mathrm{E}$ to $130^{\circ} \mathrm{E}$ with an expected positioning accuracy of $20 \mathrm{~m}$ over the Indian ocean (Sarma et al, 2010; Ganeshan, 2012; Saikiran and Vikram, 2013).

\section{IRNSS L5 Single-Point Positioning (SPP)}

In this section we formulate the SPP model of observation equations and analyse the observability of the current IRNSS constellation for several Australian locations in its secondary service area.

\subsection{Single-epoch pseudorange-only SPP model}

We assume that a single receiver $r$ is tracking $m$ IRNSS satellites on frequency L5. The single-epoch, single-frequency linearised SPP observation equations can then be formulated in vector-matrix form as

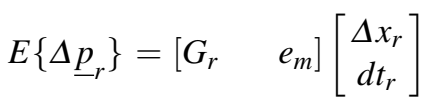

$D\left\{\Delta \underline{p}_{r}\right\}=\sigma^{2} W_{r}^{-1}$

where $E\{$.$\} and D\{$.$\} denote the expectation and disper-$ sion operators, respectively. The $m$-vector $\Delta \underline{p}_{r}$ contains the observed-minus-computed pseudorange observables to the $m$ IRNSS satellites, and underscore is used to indicate its random nature. They are a priori corrected for the ionospheric delays using the Global Ionosphere Map GIM (CODE, 2015) and for the dry tropospheric delays using the Saastamoinen model (Saastamoinen, 1972).

The $m \times 3$ matrix $G_{r}=\left[-u_{r}^{1}, \ldots,-u_{r}^{m}\right]^{T}$ contains the unit direction vectors $u_{r}^{i}, i=1, \ldots, m$, from the receiver antenna to the $m$ tracked IRNSS satellites and $e_{m}$ is the $m$-vector of ones. The 3-vector $\Delta x_{r}$ is the receiver position increment, 


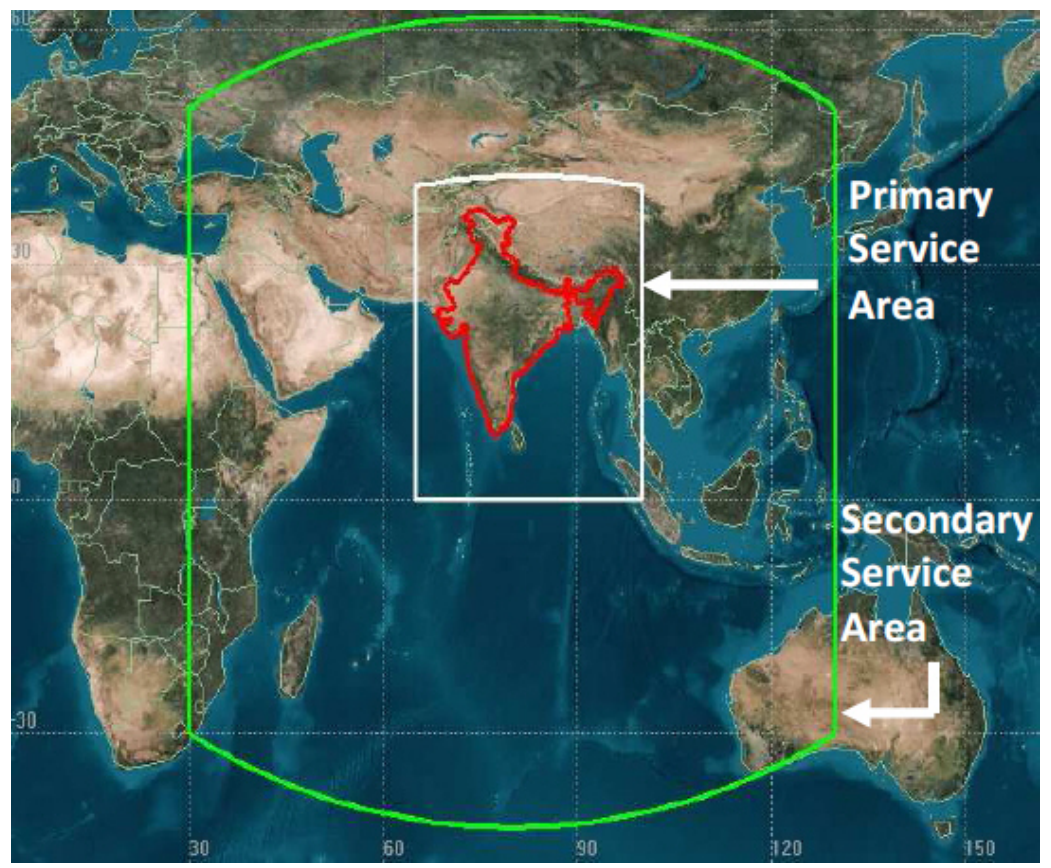

Fig. 3 IRNSS Primary and Secondary Service Area (image credit: ISRO)

and the scalar $d t_{r}$ is the increment of the receiver clock error biased by the receiver code hardware delay.

The parameter $\sigma$ denotes the zenith-referenced user range accuracy which captures the observables noise characteristics as well as the remaining unmodelled effects. The $m \times m$ diagonal weight matrix $W_{r}=\operatorname{diag}\left(w_{r}^{1}, \ldots, w_{r}^{m}\right)$ contains the elevation-dependent weights (Odolinski et al, 2014),

$w_{r}^{s}=\left(\frac{1.02}{\sin \varepsilon_{r}^{s}+0.02}\right)^{-2}$

with $\varepsilon_{r}^{s}$ being the elevation of the satellite $s$ with respect to receiver $r$.

Assuming that the design matrix $\left[G_{r}, e_{m}\right]$ is of full rank, the SPP weighted least-squares solution of the position vector is given as

$\Delta \hat{x}_{r}=\left(G_{r}^{T} W_{D} G_{r}\right)^{-1} G_{r}^{T} W_{D} \Delta p_{r}$

$Q_{\hat{x}_{r} \hat{x}_{r}}=\sigma^{2}\left(G_{r}^{T} W_{D} G_{r}\right)^{-1}$

in which $W_{D}=D_{m}\left(D_{m}^{T} W_{r}^{-1} D_{m}\right)^{-1} D_{m}^{T}$ and the range space of the $m \times(m-1)$ difference matrix $D_{m}$ spans the orthogonal complement of $e_{m}$, i.e. $D_{m}^{T} e_{m}=0$. Note that $\Delta p_{r}$ of (3) is a realization of the random observable $\Delta \underline{p}$.

\subsection{Rank-defect receiver-satellite geometries}

As the IRNSS system is currently still in a built-up phase, one can expect that there will be occasions of poor receiver satellite geometries. To understand what they look like, we now give a geometric interpretation to the occurrence of a rank-defect in the $m \times 4 \mathrm{SPP}$ design matrix $\left[G_{r}, e_{m}\right]$. The design matrix is rank defect if linear combinations of its column vectors exist that equal the zero vector, i.e. if a vector $d \in \mathbb{R}^{3}$ and a scalar $\lambda \in \mathbb{R}$ can be found such that

$G_{r} d+e_{m} \lambda=0$

As the columns of $-G_{r}^{T}$ are given by the receiver-satellite unit direction vectors $u_{r}^{s}, s=1, \ldots, m$, we may write (4) also as $\left(u_{r}^{s}\right)^{T} d=\lambda$ for $s=1, \ldots, m$, or, by using the cosine-rule with const $=\lambda /\|d\|$, as

$\cos \angle\left(u_{r}^{s}, d\right)=$ const for $s=1, \ldots, m$

Hence, the design matrix is rank defect if the unit direction vectors of the satellites $u_{r}^{s}$ all have the same constant angle to vector $d$. Geometrically this means that this rank deficiency occurs when all these unit direction vectors lie on a cone having $d$ as its symmetry axis (see Figure 4(a)). The symmetry axis of the cone, i.e. the vector $d$, is then the direction in which the position solution has become indeterminate. Thus if $d$ points towards the zenith, as in Figure 4(a) for example, then it is the height component that has become indeterminate. Figure 4(b) shows a skyplot example in which the receiver-satellite direction vectors all lie on a cone with symmetry axis having an azimuth of $300^{\circ}$ and an elevation of $60^{\circ}$.

Another case of (5) which is relevant for IRNSS is when const $=0$. In that case all receiver-satellite direction vectors $u_{r}^{i}$ lie in a plane with $d$ as its normal vector. Thus if, for example, all satellites lie in an east-west oriented plane, then the indeterminate direction vector $d$ would point in the 


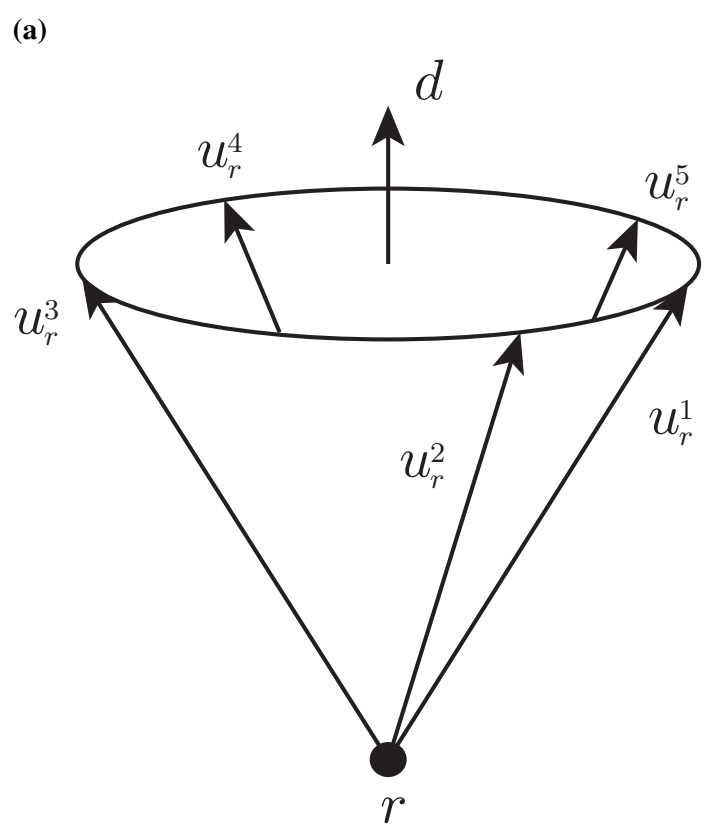

(b)

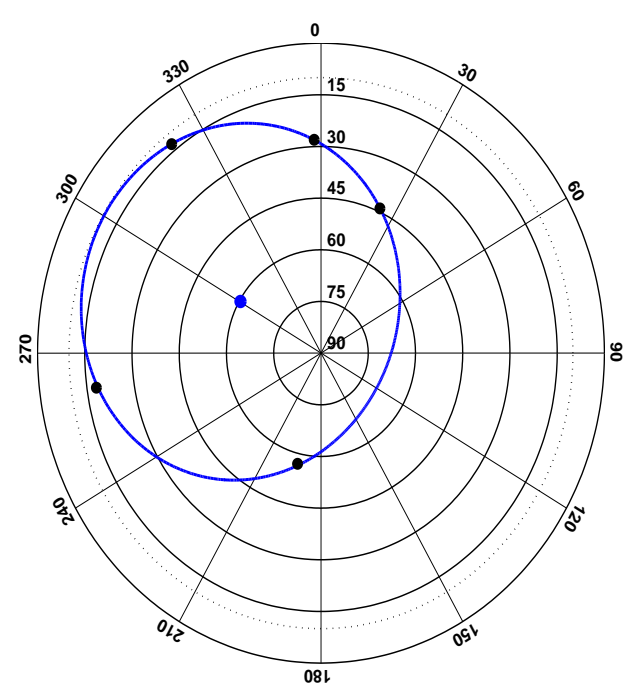

Fig. 4 (a) SPP positioning is indeterminate in direction $d$ if the receiver-satellite direction vectors $u_{r}^{s}, s=1, \ldots, m$, all lie on a cone having $\mathrm{d}$ as its symmetry axis (Teunissen, 1990). (b) Skyplot showing a cone with symmetry axis $d$ having an azimuth of $300^{\circ}$ and an elevation of $60^{\circ}$.

north-south direction. Hence, if the receiver-satellite geometry is such that the geometric extent is smallest in northsouth direction, then it is also that direction in which positioning will perform most poorly. This may happen with IRNSS for instance, if the IGSO satellites are closest to the equatorial plane in which the GEO satellites already reside.

\subsection{Current satellite visibility}

The current constellation consists of three IGSO's and one GEO satellite. Their visibility is depicted in Figure 5 for three different Australian locations, Christmas Island, Perth and Darwin, on DOY 166 of 2015 using cut-off elevation angles of $5^{\circ}$ and $10^{\circ}$. The number of satellites that can be tracked from these three locations is shown in Figure 5(a), (c) and (e), while Figure 5(b), (d) and (f) show the corresponding 24-h IRNSS ground tracks for a cut-off elevation angle of $10^{\circ}$.

As Figure 5 shows, the number of current satellites that can be tracked reduces the more eastward the Australian user is located. Although still four satellites can be still tracked on a continuous basis at Christmas island, there are periods in Perth and Darwin when this number drops to three and in Darwin even to two. Although the durations of these periods can be shortened somewhat by using $5^{\circ}$ instead of $10^{\circ}$ cut-off elevations, they remain present when tracking from Perth and Darwin.

To remedy the situation that continuous SPP positioning is currently not possible at these locations, we investigate what the inclusion of a soft height constraint in the SPP model brings in terms of its positioning capability. Assuming that the geodetic height $h_{r}$ of the receiver antenna is known with a certain standard deviation $\sigma_{h_{r}}$ and that $\Delta x_{r}$ is represented in the local East-North-Up (ENU) frame, the SPP model (1) is extended with

$E\left\{\Delta \underline{h}_{r}\right\}=c^{T} \Delta x_{r}, D\left\{\Delta \underline{h}_{r}\right\}=\sigma_{h_{r}}^{2}$

in which $c$ denotes the canonical unit 3-vector, having 1 as its third element. With the inclusion of such weighted constraint, SPP becomes possible in principle with only three tracked satellites.

\subsection{PDOP and the receiver-satellite geometry}

We use the position dilution of precision (PDOP) as a scalar measure to diagnose the strength of the receiver-satellite geometry. The PDOP is defined as (Teunissen, 1998; HofmannWellenhof et al, 2013)

$$
\mathrm{PDOP}=\operatorname{trace}\left(\left[G_{r}^{T} W_{D} G_{r}\right]^{-1}\right)^{0.5}
$$

where $W_{D}=D_{m}\left(D_{m}^{T} W_{r}^{-1} D_{m}\right)^{-1} D_{m}^{T}$. For the weighted heightconstrained case, matrices $G_{r}$ and $W_{D}$ are adopted to take (6) into account. The corresponding 24-h PDOP time series are given in Figure 6 for Christmas Island, Perth and Darwin for DOY 166 of 2015. Note that the signatures of these time series are representable for any day as the receiver-satellite geometry repeats itself every day four minutes earlier w.r.t the previous day. 
(a)

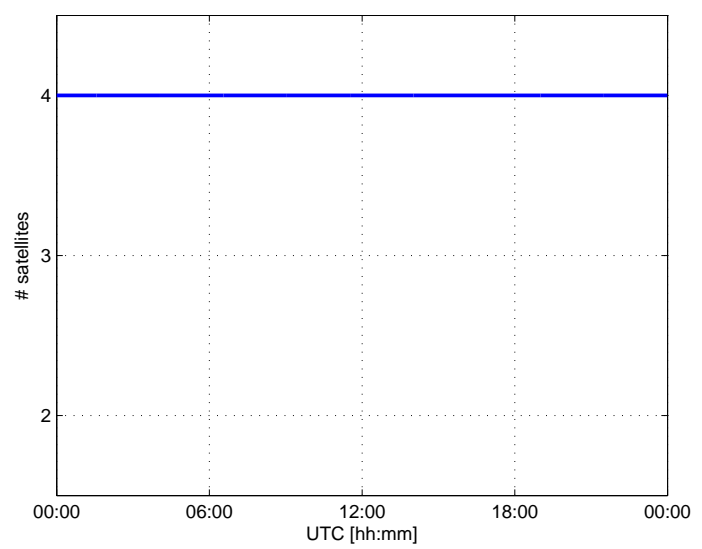

(c)

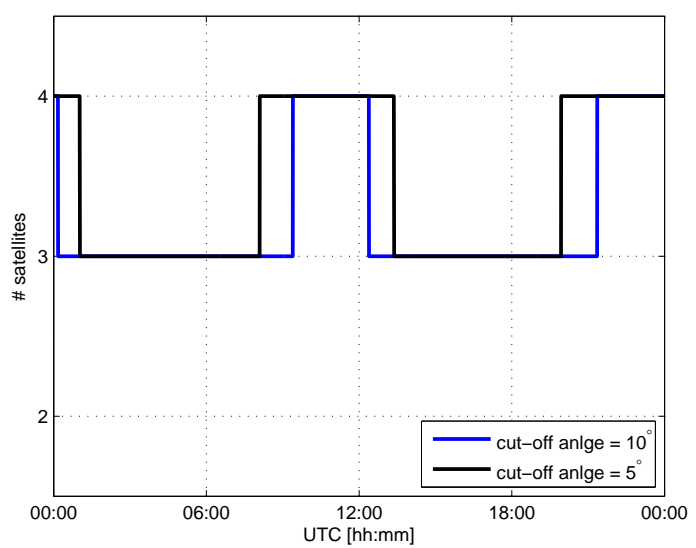

(e)

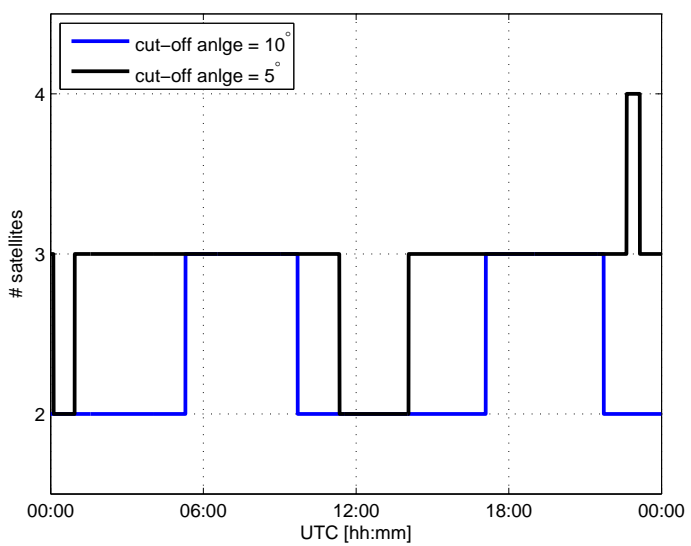

(b)

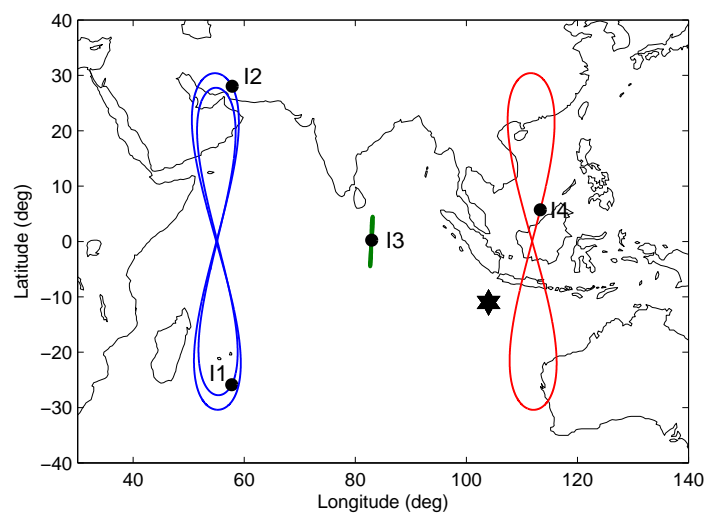

(d)

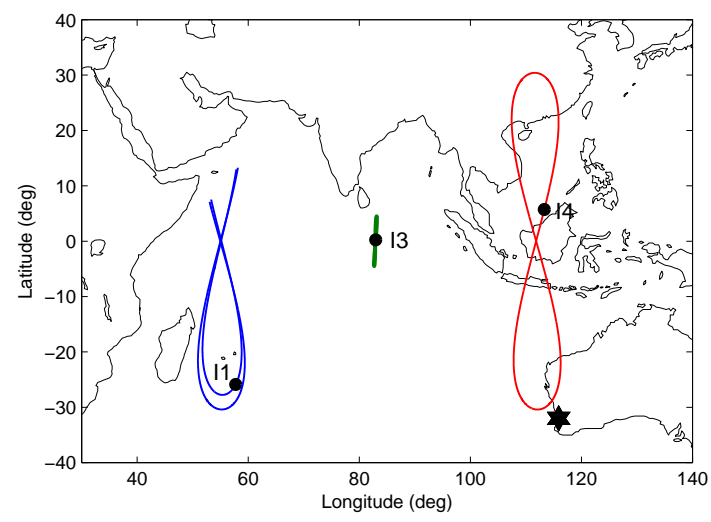

(f)

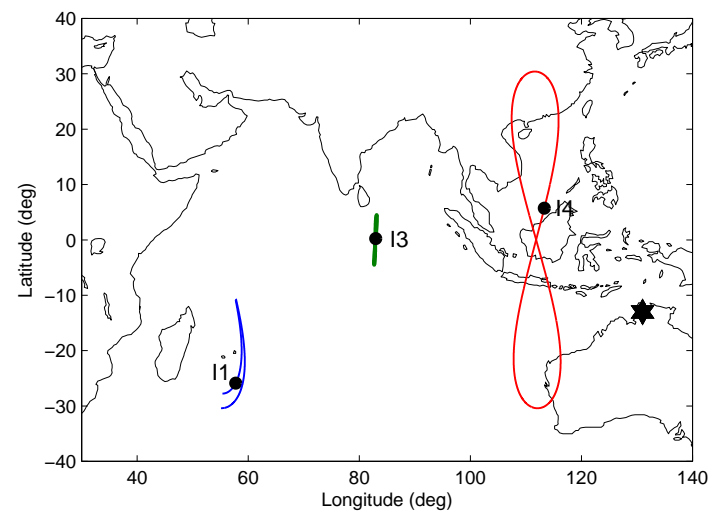

Fig. 5 Current IRNSS satellite visibility at Christmas Island, Perth and Darwin. (a) Number of satellites that can be tracked at Christmas Island, (c) at Perth and (e) at Darwin on DOY 166 of 2015, using cut-off elevation angles of $5^{\circ}$ and $10^{\circ}$, respectively. (b), (d) and (f) Corresponding satellite ground tracks on DOY 166 of 2015 for $10^{\circ}$ cut-off elevation angle. The GEO I3 ground track is shown in green, the I1 and I2 IGSOs (figures-of-eight) ground tracks are shown in blue, and the I4 IGSO ground track is shown in red . The satellite positions at UTC 06:40 are indicated by dots. The location of the users are indicated by the star symbol.

As Figure 6 shows, the PDOPs become larger, and thus the receiver-satellite geometry weaker, when one changes location from Christmas Island to Perth and then to Darwin. The PDOPs of Darwin are only shown for the two pe- 
(a)

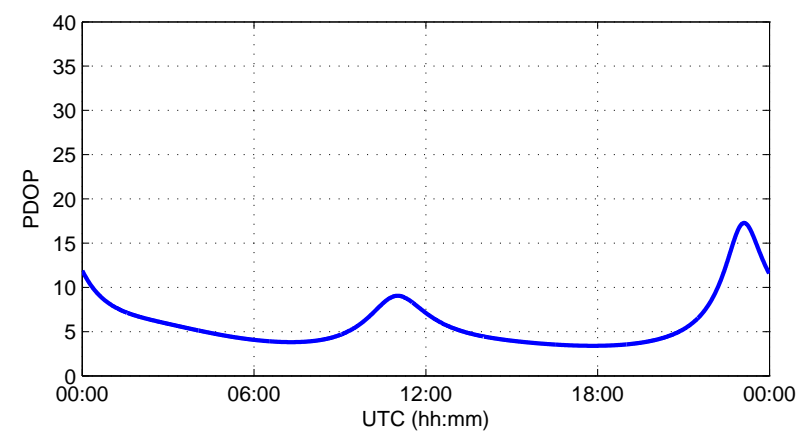

(b)

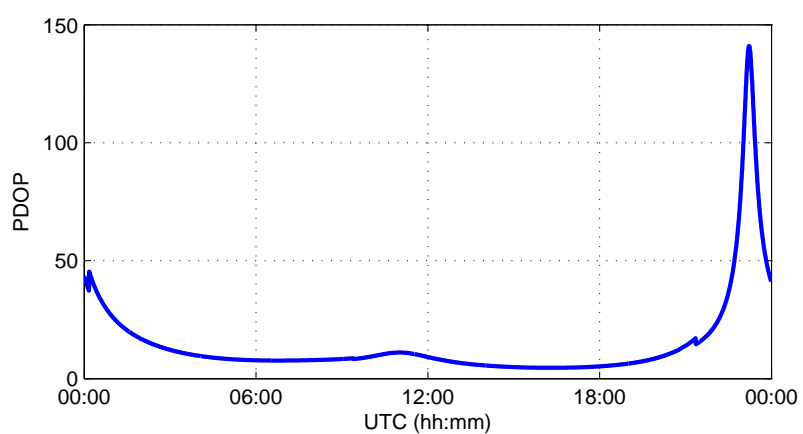

(c)

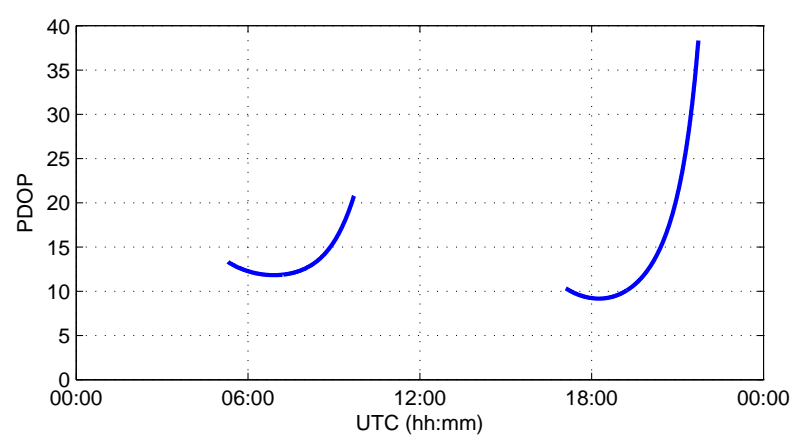

Fig. 6 Height constrained PDOPs on DOY 166 of 2015 for (a) Christmas Island, (b) Perth, and (c) Darwin, with $10^{\circ}$ cut-off elevation.

riods that three satellites are visible (see Figure 5). Note that the PDOP time series of Christmas Island is relatively flat except for two distinct peaks that built up in the UTC periods 09:16:00 - 13:18:00 and 20:51:00 - 00:00:00. They can also be recognised in the Perth PDOP time series, albeit in a somewhat distorted way. Prior to the peaks in the Perth PDOP times series, the PDOP shows a sudden drop in value due to the inclusion of a fourth satellite at UTC times 09:23:30 and 21:21:00 (see Figure 5). Thus although one is observing the maximum number of available satellites in those two periods in Perth, the receiver-satellite geometry is apparently such that it still weakens the model, thereby increasing the PDOP values.

To get a better understanding of this phenomenon, we analyse the Perth receiver-satellite geometry for the UTC period 21:21:00 - 00:04:00, which is 05:21:00 - 08:04:00 local time. Figure 7(a) shows the skyplot of the IRNSS satellites for this period. The peak in the Perth PDOP time series of Figure 6 occurs when the two satellites I1 and I2 occupy the same skyplot position. The corresponding skyplot of this instant is shown in Figure 7(b). In it we have shown the direction $d$ (cf. 4 and 5), indicated as a blue dot in the north-westerly direction, in which the receiver position is the poorest estimable, i.e. has largest variance, and two contour lines of unit vectors that make a $36^{\circ}$ resp. $38^{\circ}$ angle with this poorest estimable direction. As the skyplot shows, the receiver-satellite direction vectors of all the four satellites are on or near these contour lines, thus showing that the receiver-satellite geometry comes close to the rank-defect geometry as described in previous subsections.

Another way to illustrate this poor position estimability is as follows. As the direction vector $d$ lies in the horizontal plane (due to the imposed height constraint), the projections onto the horizontal plane of the unit vectors on the 36 resp. 38 degree cones will form a straight line. Figure 7(c) shows the projections onto the horizontal plane of the actual receiver-satellite direction vectors at 07:13:00 Perth local time on DOY 166. The fact that they all lie on or close to a straight line again indicates that the corresponding design matrix in this period is near singular.

\section{IRNSS SPP Precision}

In this section we analyse, formally as well as empirically, the SPP precision that can be achieved with the current constellation.

\subsection{Noise characteristics}

To analyse the SPP precision we first need a representative value for the zenith-referenced $\sigma^{2}$ of (1). The estimation of $\sigma^{2}$ can be done by means of variance component estimation (VCE) provided redundancy is present (Teunissen and Amiri-Simkooei, 2008). To create redundancy for the present constellation, we use data from a tracking station of which the coordinates are known. With the receiver coordinate vector $x_{r}$ known, the redundancy equals $m-1$, thus providing single-epoch redundancy for two or more satellites. With the receiver position known, the single-epoch misclosure vector of the SPP model becomes

$\underline{s}=D_{m}^{T} \Delta \underline{p}_{r}$

having zero expectation, $E\{\underline{s}\}=0$ and dispersion $D\{\underline{s}\}=$ $\sigma^{2} D_{m}^{T} W_{r}^{-1} D_{m}$. The estimated variance component based on the misclosure vector of epoch $i$ follows then as

$\underline{\hat{\sigma}}^{2}(i)=\frac{\underline{s}^{T}(i) C_{s}^{-1}(i) \underline{s}(i)}{\left(m_{i}-1\right)}$ 
(a)

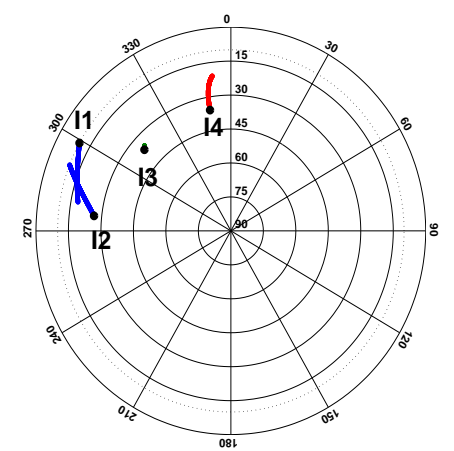

(b)

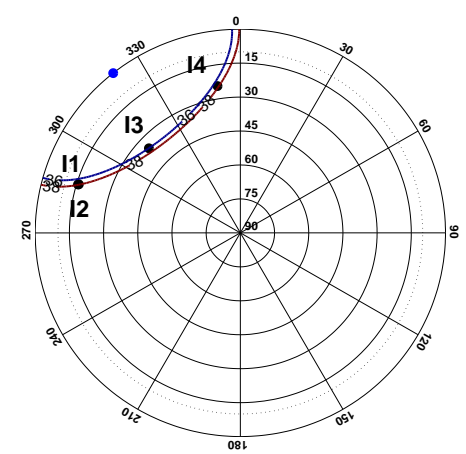

(c)

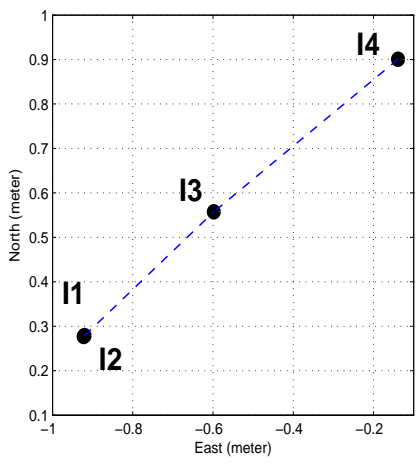

Fig. 7 (a) IRNSS skyplot for Perth, on DOY 166 of 2015 during [05:21:00-08:04:00] Perth local time. The black dots show the location of the visible satellites at the first epoch of the mentioned time interval. (b) IRNSS skyplot for Perth, at 07:13:00 Perth local time on DOY 166 of 2015. The blue dot illustrates the direction $d$ along which the receiver position is poorest estimable. The two colored contour lines show the loci of the unit vectors that make the same 36 resp. 38 degree angle with $d$. (c) The projections onto the horizontal plane of the four Perth receiver-satellite direction vectors lie approximately on a straight line at 07:13:00 Perth local time on DOY 166 of 2015.

with $C_{s}(i)=D_{m_{i}}^{T} W_{r}^{-1}(i) D_{m_{i}}$. We used this estimator to determine a $\hat{\sigma}(i)$ time series. A 10-day data set was used, collected by CUT3, a static JAVAD TRE_G3TH_8 receiver at Curtin University, employing a $30 \mathrm{~s}$ sampling rate and a $10^{\circ}$ cut-off elevation angle. The so obtained $\hat{\sigma}(i)$ time series is shown in Figure 8(b) for DOY 200 of 2015.

For the relatively crude pseudorange-only SPP model, the estimates $\hat{\sigma}(i)$ will include all remaining unmodelled effects, such as e.g., satellite orbital errors, satellite clock errors and residual atmospheric delays. However, the $\hat{\sigma}(i)$ time series of Figure 8(b) also shows some peculiar jumps. We were able to trace these jumps back to the performance of satellite I4, in particular to its on-board frequency. Figure 8 (a) shows the time series of the I4 satellite clock correction provided by the broadcast ephemerides on DOY 200 of 2015. By means of the vertical dashed lines, highlighting the jumps, it can be observed that most of the jumps in the clock time series correspond to those of the $\hat{\sigma}(i)$ time series. This finding of the jumps in the on-board frequency of satellite I4 is consistent with that of (Babu et al, 2015).

After having removed the impact of satellite I4 from the data-set, the corresponding jumps in the $\hat{\sigma}(i)$ time series disappeared and an average value of $1 \mathrm{~m}$ was obtained. This is the value that we used for our SPP positioning precision analysis.

\subsection{SPP positioning precision: formal and empirical}

We now determine the instantaneous SPP positioning precision that can be achieved with the current constellation by the use of a data-set collected on DOYs 182, 195, 200, 201, 205 , and 207 of 2015 at the rate of $30 \mathrm{~s}$ and with the cut-off elevation angle of $10^{\circ}$. This cannot however be captured by using a single averaged precision value, since the receiver- (a)

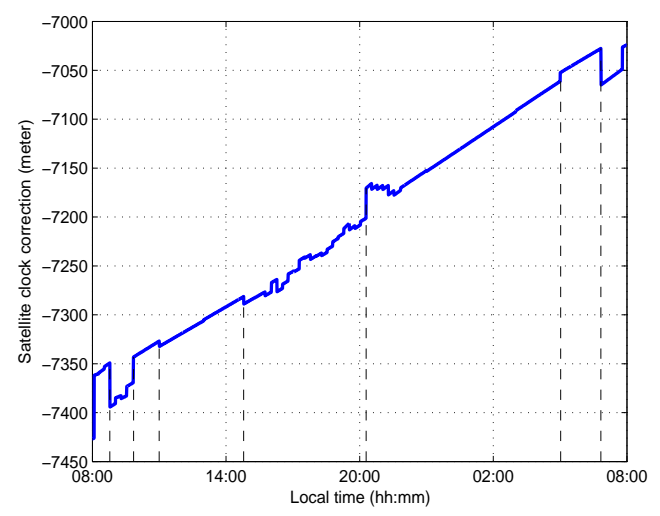

(b)

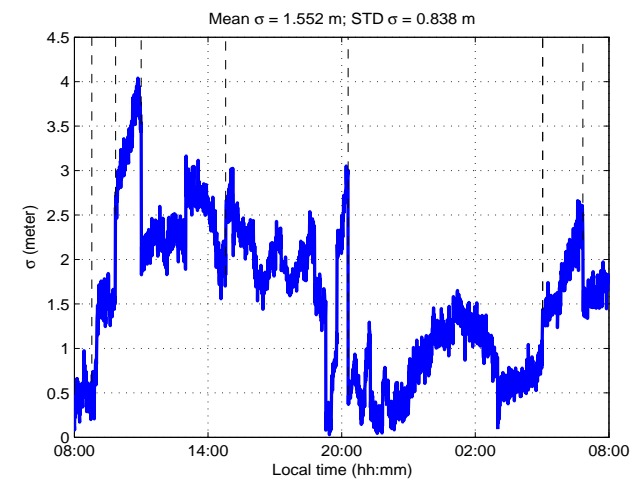

Fig. 8 (a) Time series of the I4 satellite clock corrections as provided by the broadcast ephemerides on DOY 200 of 2015. (b) The $\hat{\sigma}(i)$ time series as obtained from the CUT3 data on DOY 200 of 2015 with $10^{\circ}$ cut-off elevation.

satellite geometry, as observed in Perth, changes rather significantly over a $24 \mathrm{~h}$ period. Therefore, to illustrate this, we will work with three different time windows: 
(a)

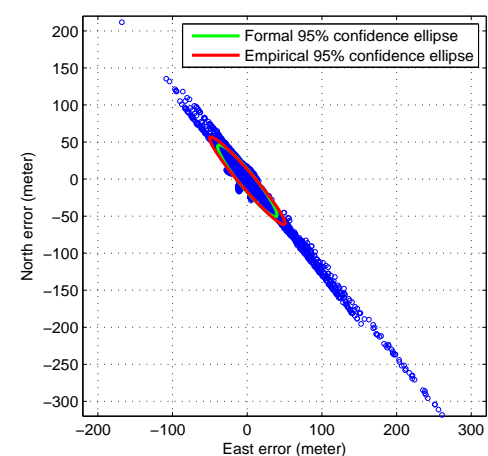

(b)

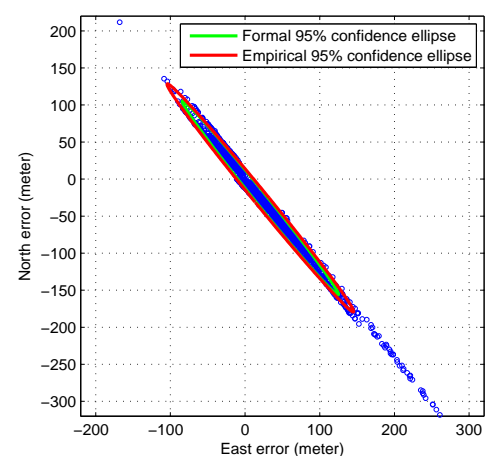

(c)

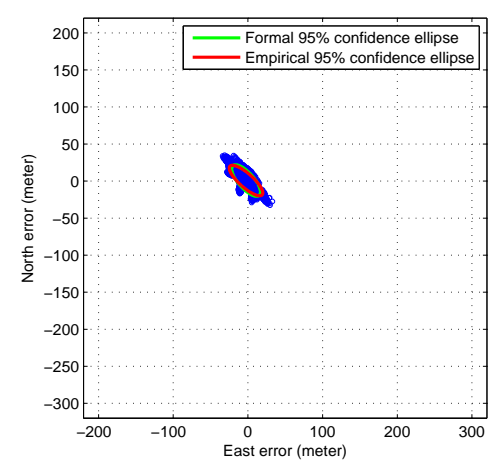

Fig. 9 IRNSS height-constrained SPP horizontal scatter plots $\left(h_{r}=24 \mathrm{~m}\right.$ and $\left.\sigma_{h_{r}}=1 \mathrm{~m}\right)$ for time window 1 (a), time window 2 (b), and time window 3 (c), based on $10^{\circ}$ cut-off elevation CUT3 data collected on DOYs 182, 195, 200, 201, 205 and 207 of the year 2015.

1. time window 1: this covers the whole $24 \mathrm{~h}$ of a day;

2. time window 2: this covers the local time period 04:19:30 - 07:02:30 of DOY 182 and its counterparts on the other days;

3. time window 3: this covers time window 1 with the exclusion of time window 2.

Note that the receiver-satellite geometries in time window 2 are the same as those during 05:21:00 - 08:04:00 on DOY 166 (cf. Figure 7). In Figure 9, the scatter plots of the positioning errors are shown for the time windows 1, 2 and 3, along with their empirical and formal $95 \%$ confidence ellipses. Note that the scatter plots are elongated in the northwesterly direction, which conforms to the direction of the symmetry axis of the cone shown in Figure 7. Note that the first two scatter plots of Figure 9 are quite similar, whereas the third is much smaller in extent. The fact that the first two scatter plots do not differ by much in extent is due to the overwhelming influence of the poor receiver-satellite geometry of the time window 2 . The scatter plot of time window 3 is much smaller as it corresponds to periods of much better receiver-satellite geometries, see also Figure 6.

Table 1 lists the formal and empirical standard deviations for different values of $\sigma_{h_{r}}$ using the data of the mentioned six DOYs. The formal standard deviations are obtained from taking the average of all the single-epoch leastsquares position variance matrices. The empirical standard deviations are obtained from the differences of the estimated SPP positions and the available ground truth of CUT3.

The results of Table 1 show that the best precision is achieved in time window 3 , while the worst is achieved in time window 2, which indeed is the period in which the receiver-satellite geometry is poorest (see Figures 6 and 7). The results also show a reasonable consistency between the empirical and formal values, with the best matches being obtained for time window 3 . This consistency shows that the easy-to-compute formal values can indeed be used to predict the expected precision with which SPP positioning can
Table 1 IRNSS single-epoch SPP empirical and formal standard deviations based on L5 data collected by station CUT3 in Perth $\left(10^{\circ}\right.$ cut-off elevation), on DOYs 182, 195, 200, 201, 205 and 207 of 2015.

\begin{tabular}{|c|c|c|c|c|c|}
\hline \multirow[t]{2}{*}{ Height precision } & \multirow[t]{2}{*}{ Time Window } & \multicolumn{2}{|c|}{$\sigma_{N}(\mathrm{~m})$} & \multicolumn{2}{|c|}{$\sigma_{E}(\mathrm{~m})$} \\
\hline & & emp & form & emp & form \\
\hline \multirow{3}{*}{$\sigma_{h_{r}}=1 \mathrm{~m}$} & 1 & 24.16 & 19.81 & 20.62 & 16.23 \\
\hline & 2 & 62.94 & 53.45 & 51.07 & 42.74 \\
\hline & 3 & 8.41 & 8.67 & 9.17 & 7.88 \\
\hline \multirow{3}{*}{$\sigma_{h_{r}}=5 \mathrm{~m}$} & 1 & 25.26 & 21.04 & 21.55 & 17.37 \\
\hline & 2 & 66.67 & 56.86 & 53.77 & 45.33 \\
\hline & 3 & 8.93 & 9.13 & 9.92 & 8.71 \\
\hline \multirow{3}{*}{$\sigma_{h_{r}}=10 \mathrm{~m}$} & 1 & 27.84 & 24.02 & 23.66 & 20.17 \\
\hline & 2 & 75.46 & 64.93 & 60.20 & 51.47 \\
\hline & 3 & 9.85 & 10.41 & 11.34 & 10.87 \\
\hline
\end{tabular}

Note: emp: empirical; form: formal; time window 1: outcomes based on all the observations; time window 2 : outcomes based on the observations made during the local time period 04:19:30-07:02:30 on DOY 182 and its counterparts on other days; time window 3: outcomes based on time window 1 with the exclusion of time window 2 .

be achieved. In summary, the results of Table 1 show that a single-epoch, pseudorange-only positioning standard deviation in North and East of about $10 \mathrm{~m}$ can be achieved with the current constellation.

\section{IRNSS Relative-Point Positioning (RPP)}

In this section we discuss the IRNSS current relative-point positioning performance for both the pseudorange-only case and the phase-smoothed case. 
(a)

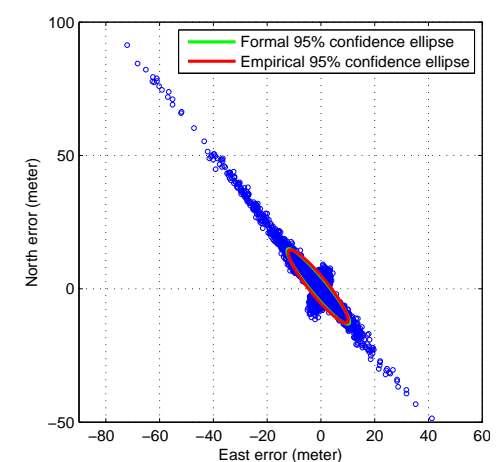

(b)

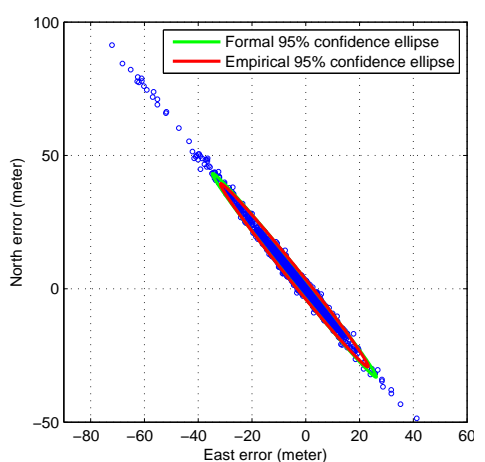

(c)

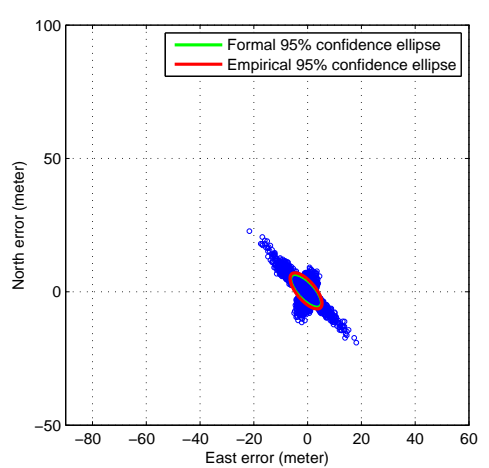

Fig. 10 IRNSS height-constrained RPP horizontal scatter plots $\left(\sigma_{h_{12}}=1 \mathrm{~m}\right)$ for time window 1 (a), time window 2 (b), and time window 3 (c), based on $10^{\circ}$ cut-off elevation using SD pseudorange data of CUT3 and CUBB collected on DOYs 182, 195, 200, 201, 205 and 207 of the year 2015.

(a)

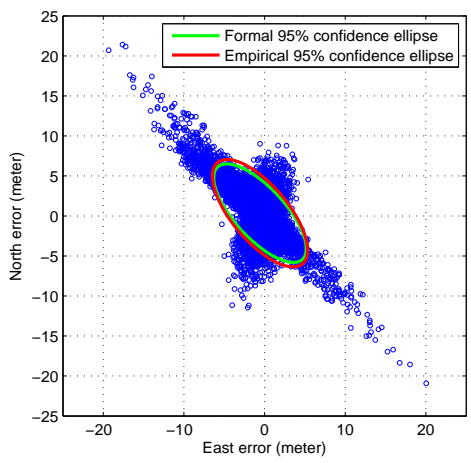

(b)

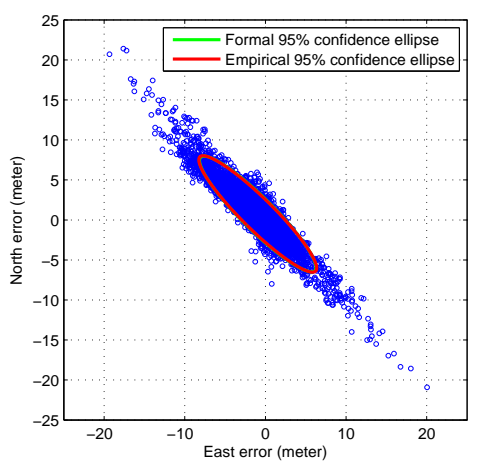

(c)

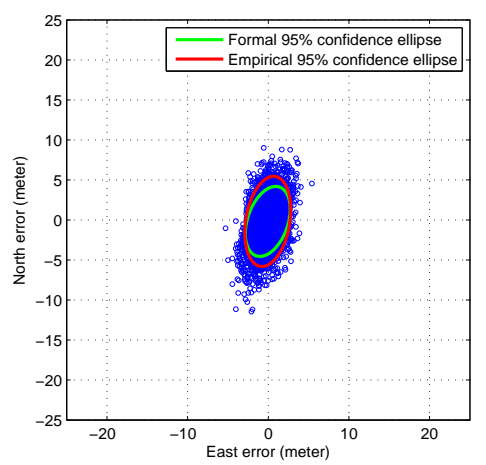

Fig. 11 IRNSS single-frequency height-constrained single-frequency RPP $\left(\sigma_{h_{12}}=1 \mathrm{~m}\right)$ results of time window 3: (a) Whole period of time window 3, (b) [16:19:00 - 01:33:00] Perth local time on DOY 182 and its counterparts on other days, (c) Remaining part of the whole time window 3.

\subsection{Pseudorange-only single-frequency RPP}

The linearised RPP model of observation equations read

$$
E\left\{\Delta \underline{p}_{12}\right\}=\left[\begin{array}{ll}
G & e_{m}
\end{array}\right]\left[\begin{array}{l}
\Delta x_{12} \\
d t_{12}
\end{array}\right]
$$

$D\left\{\Delta \underline{p}_{12}\right\}=2 \sigma_{p}^{2} W^{-1}$

in which $(.)_{12}=(.)_{2}-(.)_{1}$ is used to denote the betweenreceiver single-differenced (SD) observations and parameters. $\Delta x_{12}$ is the between-receiver baseline increment, while $\sigma_{p}$ is the zenith-referenced pseudorange standard deviation. As the unmodelled effects common to both receivers bocome eliminated in the between-receiver differencing, a large part of the residual biases present in the SPP model are absent in the RPP model. This also implies that in the case of the RPP model, $\sigma_{p}$ mainly consists of the pseudorange measurement noise, which in our case was determined as $0.2 \mathrm{~m}$ (Nadarajah et al, 2015).

To illustrate how the RPP model succeeds in eliminating the large biases present in the SPP model, Figure 10 shows the RPP equivalent of Figure 9. A comparison between the two figures shows that the scatters have become smaller and that the large excursions in the SPP scatters of Figure 9 have disappeared. The lack of homogeneity in the scatters of Figure 10 seems to indicate that there are still some significant unmodelled effects left. This, however, is not due to the presence of biases, but rather to the significant change that the receiver-satellite geometry undergoes in these time windows. We have illustrated this in Figure 11. This figure shows the time window 3 scatter (a), split out in a scatter for the local time window 16:19:00 - 01:33:00 on DOY 182 and its counterparts in other days (b), and the remaining period of time window 3 (c). Due to the significant change in receiver-satellite geometry, we indeed see the two quite different scatter signatures in the last two scatters of Figure 11 . 
Table 2 IRNSS single-epoch, pseudorange-only RPP empirical and formal standard deviations based on L5 data collected for station pairs CUT3-CUBB (emp1) and CUAA-SPA7 (emp2) in Perth (10 cut-off elevation), on DOYs 182, 195, 200, 201, 205 and 207 of 2015.

\begin{tabular}{|c|c|c|c|c|c|c|c|}
\hline \multirow[t]{2}{*}{ Height precision } & \multirow[t]{2}{*}{ Time window } & \multicolumn{3}{|c|}{$\sigma_{N}(\mathrm{~m})$} & \multicolumn{3}{|c|}{$\sigma_{E}(\mathrm{~m})$} \\
\hline & & emp1 & emp2 & form & emp1 & emp2 & form \\
\hline \multirow{3}{*}{$\sigma_{h_{12}}=1 \mathrm{~m}$} & 1 & 5.82 & 5.86 & 5.78 & 4.73 & 4.74 & 4.75 \\
\hline & 2 & 14.77 & 15.57 & 15.60 & 11.78 & 12.29 & 12.46 \\
\hline & 3 & 2.74 & 2.58 & 2.52 & 2.41 & 2.35 & 2.34 \\
\hline \multirow{3}{*}{$\sigma_{h_{12}}=5 \mathrm{~m}$} & 1 & 8.30 & 7.86 & 8.35 & 7.12 & 6.86 & 7.21 \\
\hline & 2 & 21.63 & 20.42 & 22.35 & 16.98 & 16.00 & 17.62 \\
\hline & 3 & 3.91 & 3.98 & 3.78 & 4.31 & 4.47 & 4.33 \\
\hline \multirow{3}{*}{$\sigma_{h_{12}}=10 \mathrm{~m}$} & 1 & 10.94 & 11.03 & 11.86 & 10.12 & 10.19 & 10.79 \\
\hline & 2 & 27.03 & 27.55 & 30.48 & 21.15 & 21.51 & 23.92 \\
\hline & 3 & 6.33 & 6.26 & 6.25 & 7.57 & 7.59 & 7.59 \\
\hline
\end{tabular}

Note: emp: empirical; form: formal; time window 1: outcomes based on all the observations; time window 2: outcomes based on the observations made during the local time period 04:19:30-07:02:30 on DOY 182 and its counterparts on other days; time window 3: outcomes based on time window 1 with the exclusion of time window 2

5.2 Pseudorange-only RPP positioning precision: formal and empirical

We now determine the precision that can be achieved with the current constellation using the RPP model (10). Two independent sets of data were used: one data-set was obtained from the IRNSS tracking station pair CUT3-CUBB and the other from the pair CUAA-SPA7, all of JAVAD TRE_G3TH_8 type. Both sets of data were collected on DOYs 182, 195, 200, 201, 205 and 207 of 2015 at the rate of $30 \mathrm{~s}$ with the elevation cut-off angle of $10^{\circ}$.

Table 2 lists the formal and empirical standard deviations for different values of $\sigma_{h_{12}}$ using the two data sets of the above-mentioned six DOYs. The entries in this table have been determined in the same way as the entries in Table 1. Comparison with the SPP results of Table 1 shows that the precision has improved and that the factor of improvement is indeed approximately $5 / \sqrt{2}$. The results show agreement between the empirical results ('emp1' and 'emp2' of Table 2) and also a reasonable consistency between the empirical and formal values. In summary, the results of Table 2 show that a single-epoch, L5 pseudorange-only RPP positioning standard deviation in North and East of about 7 $\mathrm{m}$ can be achieved with the current IRNSS constellation.

5.3 Phase-smoothed RPP positioning precision: formal and empirical

In this section we study the impact of replacing the raw pseudorange observations by their more precise carrier-phasesmoothed counterparts. The phase-smoothed pseudoranges, denoted here as $\bar{p}_{r}(i)$, are computed recursively from the original pseudoranges $p_{r}(i)$ and carrier-phases $\phi_{r}(i)$ as (Hatch,
Table 3 IRNSS single-epoch, phase-smoothed RPP empirical and formal standard deviations based on L5 data collected for station pair CUT3-CUBB in Perth (10 cut-off elevation), on DOYs 182, 195, 200, 201, 205 and 207 of 2015.

\begin{tabular}{|c|c|c|c|c|c|}
\hline \multirow[t]{2}{*}{ Height precision } & \multirow[t]{2}{*}{ Time window } & \multicolumn{2}{|c|}{$\sigma_{N, 50}\left[\sigma_{N, 100}\right](\mathrm{m})$} & \multicolumn{2}{|c|}{$\sigma_{E, 50}\left[\sigma_{E, 100}\right](\mathrm{m})$} \\
\hline & & emp & form & emp & form \\
\hline \multirow{3}{*}{$\sigma_{h_{12}}=1 \mathrm{~m}$} & 1 & $2.55[2.52]$ & $1.39[1.19]$ & $2.23[2.29]$ & $1.23[1.08]$ \\
\hline & 2 & $5.57[5.97]$ & $3.65[3.04]$ & $4.56[4.95]$ & $2.87[2.38]$ \\
\hline & 3 & $1.42[1.13]$ & $0.63[0.55]$ & $1.41[1.33]$ & $0.77[0.71]$ \\
\hline \multirow{3}{*}{$\sigma_{h_{12}}=5 \mathrm{~m}$} & 1 & $4.16[3.74]$ & $3.83[3.53]$ & $4.17[4.04]$ & $3.93[3.75]$ \\
\hline & 2 & $8.56[7.03]$ & $8.02[6.74]$ & $6.79[5.67]$ & $6.27[5.27]$ \\
\hline & 3 & $2.86[2.60]$ & $2.57[2.38]$ & $3.78[3.46]$ & $3.31[3.15]$ \\
\hline \multirow{3}{*}{$\sigma_{h_{12}}=10 \mathrm{~m}$} & 1 & $6.42[6.12]$ & $6.59[6.25]$ & $7.04[6.96]$ & $7.19[7.00]$ \\
\hline & 2 & $10.80[9.38]$ & $11.33[9.49]$ & $8.52[7.44]$ & $8.86[7.41]$ \\
\hline & 3 & $4.94[4.57]$ & $5.08[4.73]$ & $6.35[6.17]$ & $6.52[6.23]$ \\
\hline
\end{tabular}

Note: emp: empirical; form: formal; time window 1: outcomes based on all the observations; time window 2: outcomes based on the observations made during the local time period 04:19:30-07:02:30 on DOY 182 and its counterparts on other days; time window 3: outcomes based on time window 1 with the exclusion of time window 2 .

1983, 1986; Teunissen, 1991)

$\bar{p}_{r}(i)=\frac{1}{k} p_{r}(i)+\frac{k-1}{k}\left[\bar{p}_{r}(i-1)+\phi_{r}(i)-\phi_{r}(i-1)\right]$

with initialization $\bar{p}_{r}(1)=p_{r}(1)$. The smoothing can be done on the basis of a chosen window length $w l$. With the window length $w l$ specified, the factor $k$ in (11) is taken as

$k=\left\{\begin{array}{cc}i, & i \leq w l \\ w l, & i>w l\end{array}\right.$

Note, that if, during the period in which the IRNSS observations are being made, one of the satellites sets and rises again, then smoothing for the data of that satellite should be reinitialised by setting $i=1$ for the epoch in which the satellite rises again. This can be explained as follows. The phase integer ambiguity after satellite rise is not necessarily the same as that before satellite set. Therefore, if one makes use of the observations recorded before satellite set to smooth the code observations after satellite rise, the phase-smoothed code observables will become biased by the above-mentioned integer ambiguity differences.

Table 3 displays the phase-smoothed counterparts of the entries in Table 2 for the station pair CUT3-CUBB, and for the window lengths of $w l=50$, and $w l=100$. Upon comparing these two tables, we note that both empirical and formal precisions have improved, especially those corresponding to time window 2. However, it can also be observed that there is some discrepancy still between the empirical and formal results, especially when a harder constraint is imposed on the height of the receiver. This is due to the accumulation in the smoothing procedure of a residual multipath (Kim and Langley, 2000; Park et al, 2008; Zhao et al, 2009). This also 
Table 4 IRNSS day-differenced single-epoch, phase-smoothed RPP empirical and formal standard deviations based on L5 data collected for station pair CUT3-CUBB in Perth ( $10^{\circ}$ cut-off elevation), on DOYs 182, 195, 200, 201, 205 and 207 of 2015.

\begin{tabular}{|c|c|c|c|c|c|}
\hline \multirow[t]{2}{*}{ Height precision } & \multirow[t]{2}{*}{ Time window } & \multicolumn{2}{|c|}{$\sigma_{N, 50}\left[\sigma_{N, 100}\right](\mathrm{m})$} & \multicolumn{2}{|c|}{$\sigma_{E, 50}\left[\sigma_{E, 100]}\right](\mathrm{m})$} \\
\hline & & emp & form & emp & form \\
\hline & 1 & $1.97[1.70]$ & $1.97[1.68]$ & $1.80[1.62]$ & $1.75[1.54]$ \\
\hline \multirow[t]{3}{*}{$\sigma_{h_{12}}=1 \mathrm{~m}$} & 2 & $4.79[3.99]$ & $5.15[4.28]$ & $3.78[3.13]$ & $4.04[3.35]$ \\
\hline & 3 & $1.03[0.81]$ & $0.89[0.76]$ & $1.22[1.13]$ & $1.08[0.99]$ \\
\hline & 1 & $5.26[4.97]$ & $5.41[4.99]$ & $5.42[5.32]$ & $5.58[5.30]$ \\
\hline \multirow[t]{3}{*}{$\sigma_{h_{12}}=5 \mathrm{~m}$} & 2 & $10.65[9.40]$ & $11.30[9.50]$ & $8.33[7.35]$ & $8.83[7.43]$ \\
\hline & 3 & $3.58[3.23]$ & $3.55[3.23]$ & $4.56[4.41]$ & 4.62[4.36] \\
\hline & 1 & $9.36[8.90]$ & $9.31[8.84]$ & $10.17[9.96]$ & $10.17[9.89]$ \\
\hline \multirow[t]{2}{*}{$\sigma_{h 12}=10 \mathrm{~m}$} & 2 & $15.07[12.43]$ & $15.98[13.41]$ & $11.79[9.71]$ & $12.48[10.47]$ \\
\hline & 3 & $7.19[6.60]$ & $7.02[6.41]$ & $9.11[8.72]$ & $9.10[8.62]$ \\
\hline
\end{tabular}

Note: emp: empirical; form: formal; time window 1: outcomes based on all the observations; time window 2: outcomes based on the observations made during the local time period 04:19:30-07:02:30 on DOY 182 and its counterparts on other days; time window 3: outcomes based on time window 1 with the exclusion of time window 2 .

explains the increment of the empirical standard deviations after increasing the smoothing window length from $w l=50$ to $w l=100$.

As the IRNSS constellation repeats itself every sidereal day, the stations multipath can also be expected to repeat every sidereal day. Hence, one can expect to eliminate the effect of multipaths by differencing the observations between days associated with the same satellite geometry. Listed in Table 4 are the day-differenced counterparts of Table 3. Table 4 does indeed show not only that this differencing results in a better consistency between empirical and formal precisions, but also that the previously noticed accumulation effect for longer window lengths is now absent in the empirical standard deviations. The results of Table 4, divided by $\sqrt{2}$, are therefore representative for a multipathfree RPP positioning performance. In summary, the results show that a single-epoch, carrier-phase-smoothed pseudorange RPP positioning standard deviation in North and East of about $5 \mathrm{~m}$ can be achieved with the current IRNSS constellation.

\section{Fully operational IRNSS performance}

In this section we extend the previous analysis to the nearfuture case where the IRNSS has reached its full operational capability. The following investigations are based on the IRNSS geometry on DOY 166 of 2015. The orbits of the satellites I5, I6, and I7 are simulated. For simulation, the orbital parameters are determined such that they have respective nominated longitudes (ISRO, 2014a) and have similar ground tracks to the respective types of existing satellites.

\subsection{PDOP analysis}

We start with a full-constellation PDOP analysis of the same three Australian locations as considered in the previous sections, Christmas Island, Perth and Darwin. The satellite visibility and 24-h PDOP time series are shown in Figure 12(a), (c) and (e), while the corresponding satellite ground tracks are shown in Figure 12(b), (d) and (f). Note that the three PDOP time series each behave almost similarly in the periods UTC 00:00-12:00 and UTC 12:00-00:00. This is due to the fact that the IRNSS satellite configuration in the period UTC 00:00-12:00 is similar to that in UTC 12:00-00:00, albeit in the second period the positions of I1 and I2, and of I4 and I6, have interchanged.

The figure also shows that with a fully operational IRNSS, all seven satellites are visible from Christmas Island, but five and sometimes six from Perth, and only four to five from Darwin. Users in Perth would not be able to track the GEO I5 and most of the time also not the north-westerly IGSO (either I1 or I2), while users in Darwin additionally would lose most of the time track of the south-westerly IGSO also. With a reduction in number of satellites tracked, the PDOP value generally increases and the positioning precision decreases. With a lower cut-off elevation angle than $10^{\circ}$ the situation would improve, but not by much. For Perth, for instance, a $5^{\circ}$ cut-off elevation would not increase the maximum number of visible satellites, but it would lengthen the period that five satellites are visible somewhat.

In the Perth PDOP time series, there are two peaks visible, one at UTC 07:27 and another at 19:21. Although these peaks are not the same as shown in the earlier height-constrained PDOP time series for Perth (see Figure 6), they can again be explained by the occurrence of an approximate 'cone-like' receiver-satellite geometry. This is shown in the skyplots of Figure 13. In these two skyplots the symmetry axes of the two cones are also shown, i.e. the direction $d$ (cf. 4 and 5) along which the position is the poorest estimable (blue dot). Since these directions are near zenith in both cases, it is particularly the Up-component that is poorly estimable. The coloured contour plots in Figure 13 are the loci of unit vectors that make a constant angle with the cone's symmetry axis, i.e. with direction $d$. For the first epoch UTC 07:27, the receiver-satellite direction vectors are located on the contours with values of $58^{\circ}, 59^{\circ}, 64^{\circ}$ and $65^{\circ}$, respectively, and for the second epoch UTC 19:21, they are on the contours with values of $47^{\circ}, 48^{\circ}, 51^{\circ}$ and $52^{\circ}$, respectively. As the variability in angles of the first set is larger than that of the second set, the deviation of the receiver-satellite geometry from a conical geometry is larger at UTC 07:27 than at UTC 19:20. This is why the value of the PDOP is smaller at UTC 07:27 than at UTC 19:21.

If we compare the PDOP time series of Darwin with that of Perth, we note next to the generally much larger PDOP 
(a)

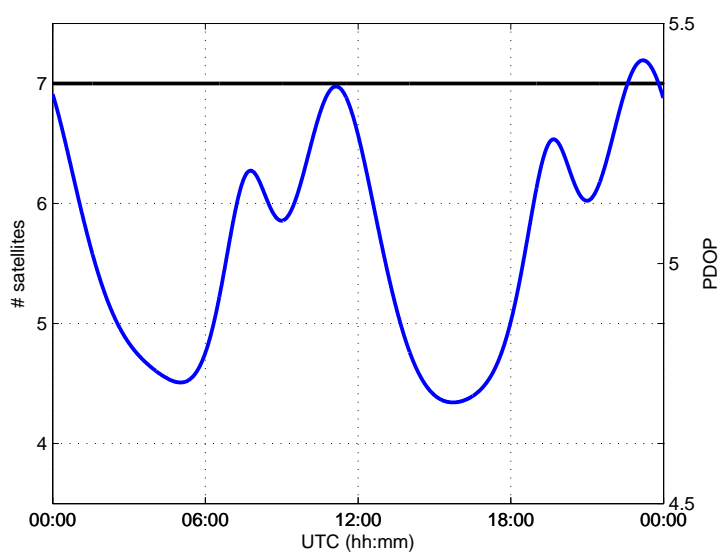

(c)

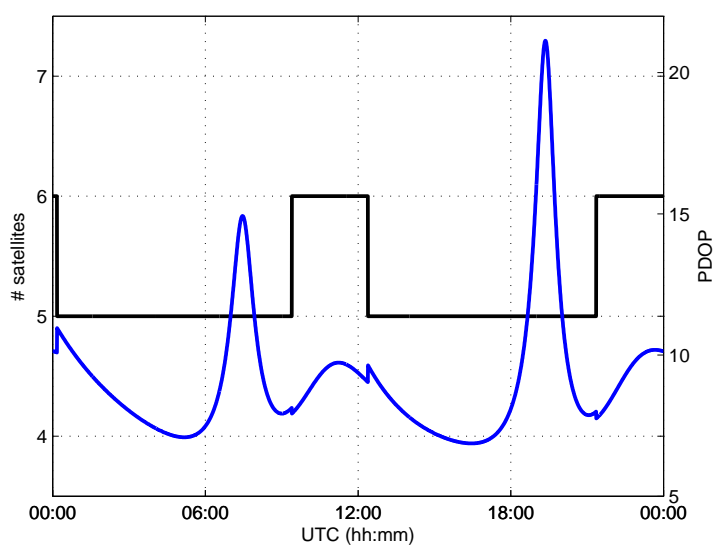

(e)

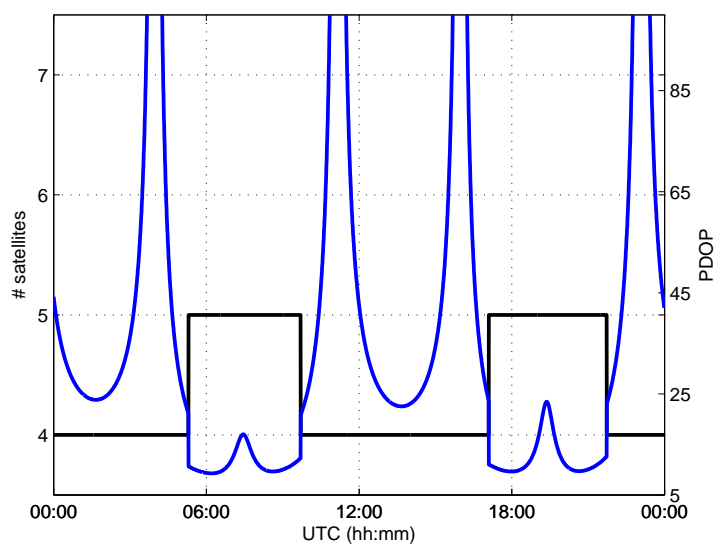

(b)

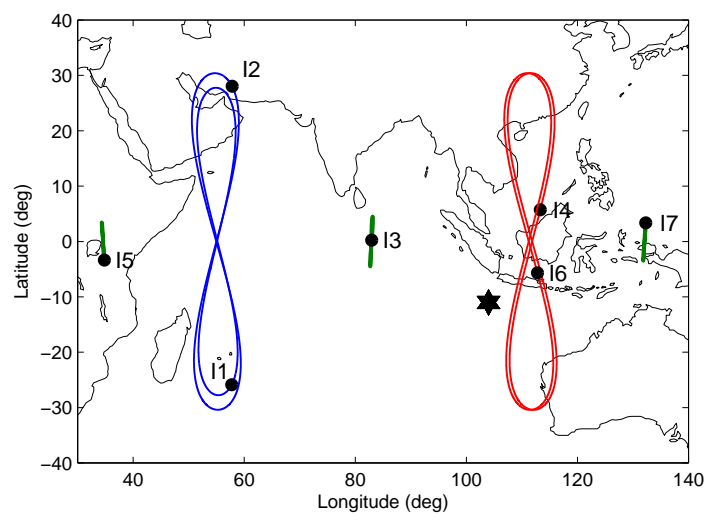

(d)

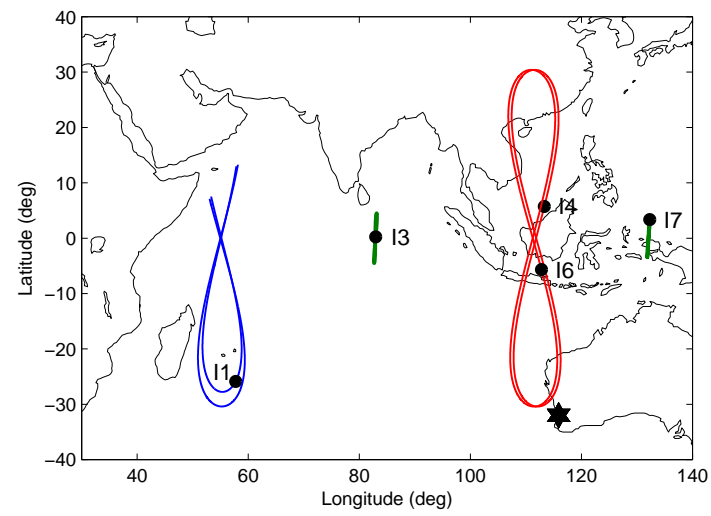

(f)

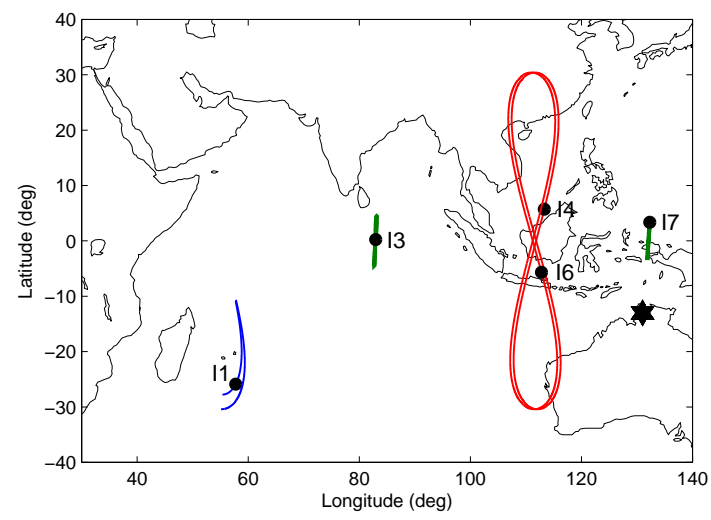

Fig. 12 Full constellation PDOPs and satellite ground tracks for Christmas Island, Perth and Darwin. (a) PDOPs and number of satellites that can be tracked at Christmas Island, (c) at Perth and (e) at Darwin, on DOY 166 of 2015, using $10^{\circ}$ cut-off elevation angle. (b), (d) and (f) Corresponding satellite ground tracks on DOY 166 of 2015 for $10^{\circ}$ cut-off elevation angle. The GEO ground tracks are shown in green, the I1 and I2 IGSOs (figures-of-eight) ground tracks are shown in blue, and the I4 and I6 IGSO ground tracks are shown in red.The satellite positions at UTC 06:40 are indicated by dots. The location of the users are indicated by the star symbol.

values in Darwin, that there are four periods when the Darwin PDOP values reach extreme values. During these four periods the receiver-satellite geometry is such that not only are a mere four satellites, namely I3, I4, I6 and I7, visi- 
(a)

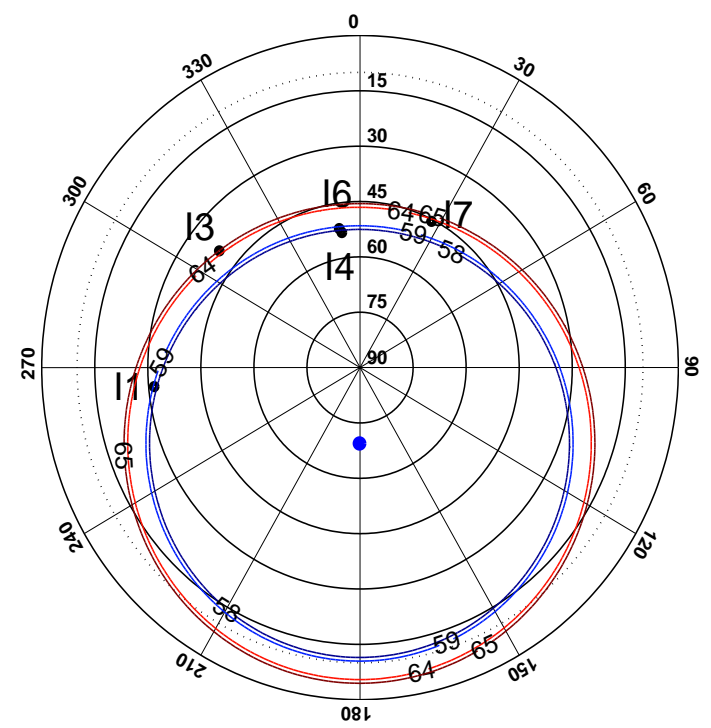

(b)

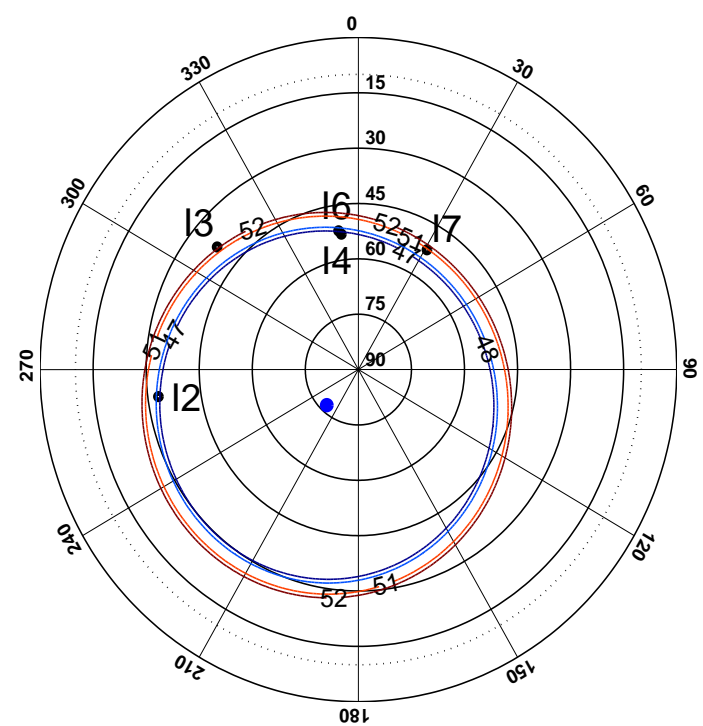

Fig. 13 (a) Fully operational IRNSS skyplot for Perth on DOY 166 of 2015 at UTC 07:27:00 (b) and UTC 19:21:00. The blue dot in each figure denotes the symmetry axis $d$ of the cone, i.e. the direction in which the receiver position is poorest estimable. The colored contour plots show the loci of unit vectors that make a constant angle with $d$.

ble, but that two of them, namely I4 and I6, also have almost the same skyplot location. Hence, since all these four receiver-satellite direction vectors will then approximately span a plane, the columns of the receiver-satellite geometry matrix $G_{r}$ of (1) will become near linearly dependent, thus leading to very large PDOP values.

\subsection{Positioning precision}

We now determine the positioning precision that one can expect to have once the full IRNSS constellation is operational. The expected single-epoch SPP precision over a whole day is shown in Figure 14 for Christmas Island, Perth and Darwin. The precision clearly deteriorates when moving eastwards, i.e. the precision is best at Christmas Island and poorest in Darwin. Each graph in Figure 14 shows three time series, one based on pseudoranges only ('non-smoothed') and another two based on carrier-phase smoothed pseudoranges ('smoothed'), with window lengths of $w l=50$ and $w l=100$, respectively (cf. 11). The average values of the time series are given on top of the graphs.

The carrier-phase smoothing clearly has a very beneficial effect on the positioning precision, particularly for Christmas Island and Perth. We see not only a significant improvement in precision of approximately one order of magnitude, but also, in the case of Christmas Island and Perth, a flattening of the time series, thus making the positioning precision almost independent of the time of day. The two peaks in the time series of the Christmas Island North standard deviation are due to a reduced extent in north-southerly direction of the satellite locations at those instances.

As Figure 14(e) and (f) show, the positioning precision at Darwin is problematic, even when carrier-phase smoothing is used. Although the carrier-phase smoothing does help to lower the standard deviations and make the durations of the peaks briefer, the carrier-phase smoothing does not eliminate the large peaks. Hence, in Darwin there will remain four periods during the day when SPP positioning better than a few meters will not be possible, even with the full IRNSS constellation operational.

The four periods that the standard deviations at Darwin will be smaller than $2 \mathrm{~m}$ are given in local time for DOY 166 as [09:58:00 , 12:11:30], [14:21:00 , 19:44:00], [22:00:30 , 00:17:00], [02:19:00, 07:38:00] for window length $w l=50$ and as [09:58:00, 12:40:30], [14:06:30, 19:59:30], [21:30:30 , 00:43:00], [02:05:00 , 07:53:30] for window length $w l=$ 100. These periods can be transferred to other days as well knowing that the IRNSS geometry repeats every day four minutes earlier. For example, if one is interested in finding the counterparts of these periods on DOY 25 of 2016, the day differences between this day and DOY 166 of 2015, which is 224 days, should be multiplied by four minutes, and then subtracted from these periods.

The above results refer to the unconstrained case. We now consider the height-constrained case. With a weighted height constraint of $\sigma_{h_{r}}=1 \mathrm{~m}$, the results of Figure $14 \mathrm{im}$ prove to those of Figure 15. These results show that positioning at Christmas Island and Perth marginally benefits from the constraint, but that Darwin, on the other hand, bene- 
(a)

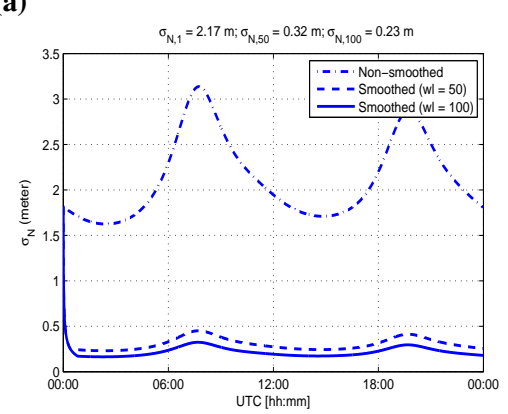

(b)

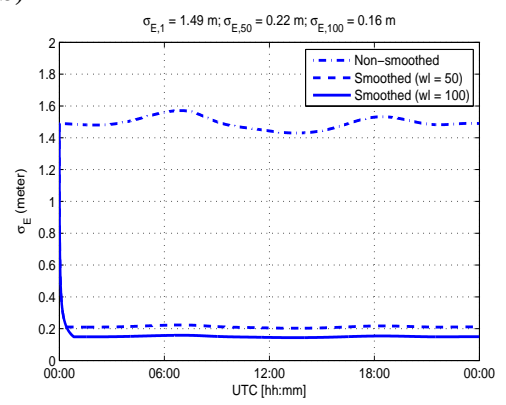

(c)

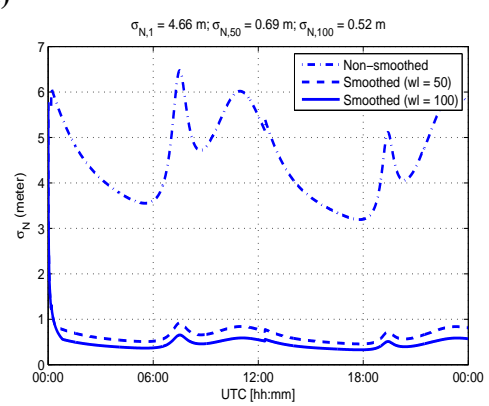

(d)

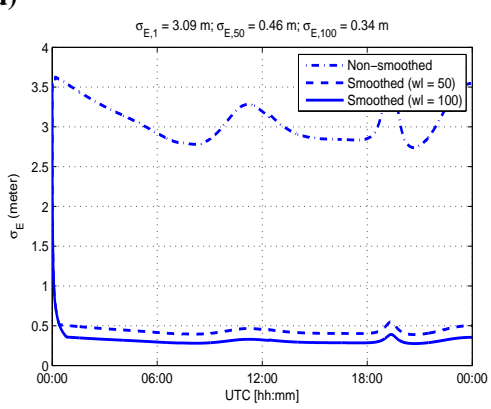

(e)

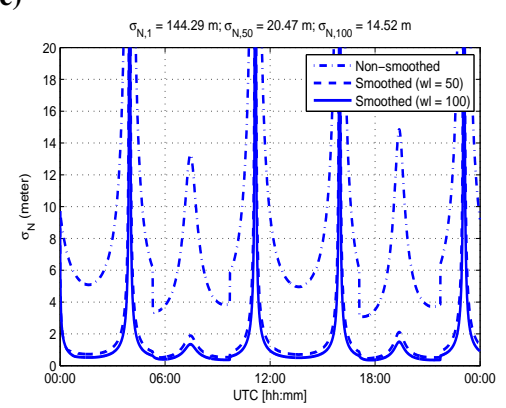

(f)

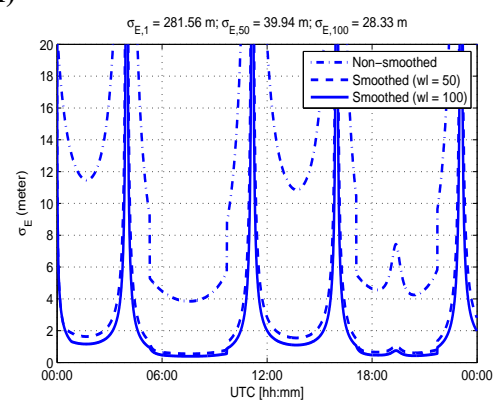

Fig. 14 Full constellation, single-epoch, horizontal SPP precision for (a) \& (b) Christmas Island (c) \& (d), Perth and (e) \& (f) Darwin. 'Nonsmoothed' is the single epoch, pseudorange-only SPP precision; 'smoothed $(w \mathrm{l}=50)$ ' is the carrier-phase-smoothed pseudorange SPP precision with window length of 50 epochs; 'smoothed $(\mathrm{wl}=100)$ ' is the carrier-phase-smoothed pseudorange SPP precision with window length of 100 epochs. Note the differences in $\sigma$-scale between the three locations. The average values of the time series are given on top of the graphs.

fits significantly. With the carrier-phase smoothing included, the positioning precision at Darwin even approaches that of Chrismas Island and Perth.

One may argue of course, that for some applications a height constraint of $\sigma_{h_{r}}=1 \mathrm{~m}$ is overly restrictive. We therefore also show the results for Darwin when the much softer constraint of $\sigma_{h_{r}}=5 \mathrm{~m}$ is used; see Figure 16. These results show that positioning at Darwin can still benefit considerably even with such a much softer constraint. With carrierphase smoothing the precision is about at the $2 \mathrm{~m}$ level, which is a dramatic improvement over the Darwin results of Figure 14.

\section{Summary}

As IRNSS will soon become fully operational, it is important to gain an understanding of its navigational potential and actual positioning capabilities. We therefore studied in this contribution the IRNSS stand-alone positioning performance for Australian stations located in the IRNSS's service area. This is the first such study performed. After a brief introduction to the IRNSS system, we described the positioning model that formed the basis for our analysis. We determined the zenith-referenced range error to be $1 \mathrm{~m}$ for SPP and $0.2 \mathrm{~m}$ for RPP. In the first part of our analysis we focussed on the currently achievable positioning per- formance. We identified and explained the occurrence of some poor receiver-satellite geometries and demonstrated with real IRNSS L5 data that instantaneous positioning better than $10 \mathrm{~m}$ for SPP and better than $7 \mathrm{~m}$ for RPP is currently feasible at the west-coast (e.g., Perth). These results are expected to improve significantly when IRNSS reaches its full operational capability. The instantaneous SPP precision for Perth was shown to improve then to $4 \mathrm{~m}$ on average and even down to $0.5 \mathrm{~m}$ if carrier-phase smoothing is included. These results get even better, due to the impact of IRNSSs receiver-satellite geometry, the more westward one moves, i.e. for marine navigation and positioning on the Indian Ocean. When moving eastwards, however, the results get poorer. At Darwin, for instance, the receiversatellite geometry is such that every day four periods exist during which the positioning precision is poor, even when carrier-phase smoothing is used. Outside these four periods, however, the positioning precision is good and comparable with that of Perth. And as demonstrated, reduction of the impact of the four periods at Darwin is possible through the inclusion of soft height constraining $\left(\sigma_{h}=5 \mathrm{~m}\right)$ combined with carrier-phase smoothing. In this case positioning at Darwin will be around $2 \mathrm{~m}$ or better for the whole day and thus still able to service a whole range of important applications. 
(a)

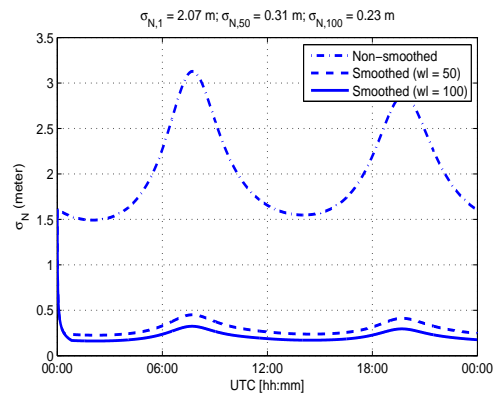

(b)

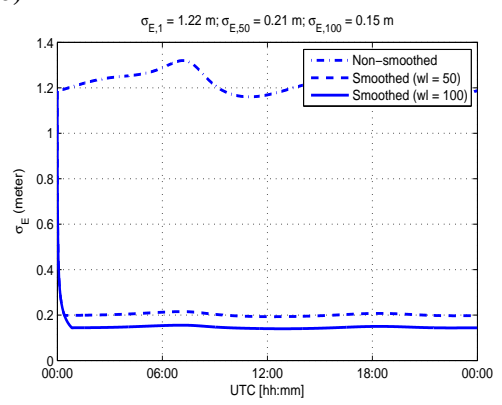

(c)

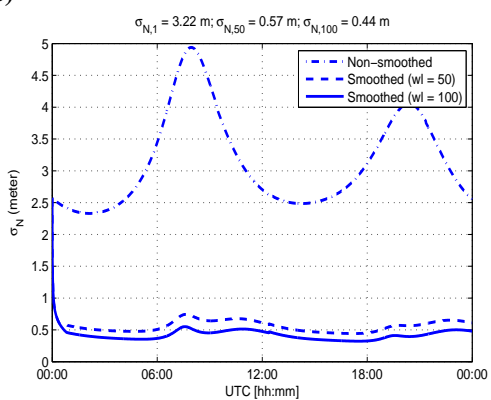

(d)

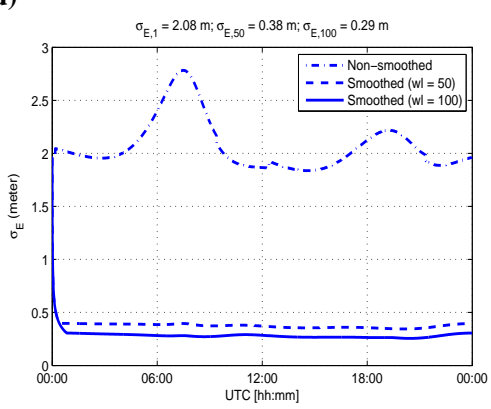

(e)

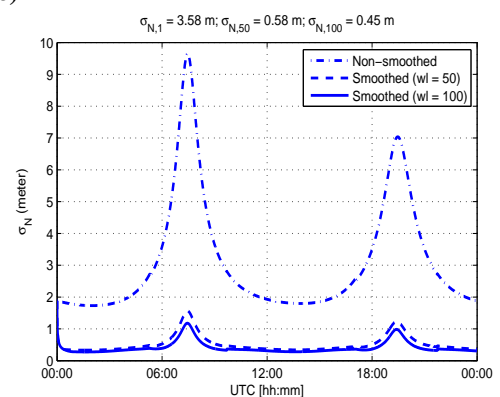

(f)

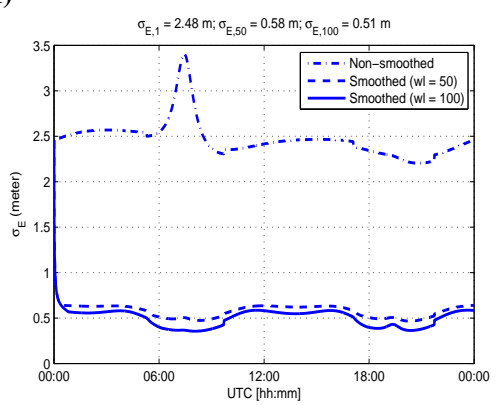

Fig. 15 Full constellation, height-constrained $\left(\sigma_{h_{r}}=1 \mathrm{~m}\right.$ ), horizontal SPP precision for (a) \& (b) Christmas Island, (c) \& (d) Perth and (e) \& (f) Darwin. 'Non-smoothed' is the single epoch, pseudorange-only SPP precision; 'smoothed (wl=50)' is the carrier-phase-smoothed pseudorange SPP precision with window length of 50 epochs; 'smoothed ( $\mathrm{wl}=100)$ ' is the carrier-phase-smoothed pseudorange SPP precision with window length of 100 epochs. Note the differences in $\sigma$-scale between the three locations. The average values of the time series are given on top of the graphs.

\section{References}

Babu R, Mula P, Ratnakara SC, Ganeshan AS (2015) IRNSS Satellite Parameter Estimation using Combination Strategy. Global Journal of Science Frontier Research 15(3)

Chandrasekhar MV, Rajarajan D, Satyanarayana G, Tirmal N, Rathnakara SC, Ganeshan AS (2015) Modernized IRNSS Broadcast Ephemeris Parameters. Control Theory and Informatics 5(2):1-9

CODE (2015) Global ionosphere maps produced by CODE. Available from : ftp://ftp.unibe.ch/aiub/CODE/2015, accessed 2 September 2015

Ganeshan AS (2012) Overview of GNSS and Indian Navigation Program. In: GNSS User Meeting, 23 Feb. 2012, ISRO Satellite Center, Bangalore

GPS World (2015) ISRO: All 7 IRNSS Satellites in Orbit by March. Available from:http://gpsworld.com/isroall-7-irnss-satellites-in-orbit-by-march, published 8 October 2015, accessed 2 November 2015

Hatch R (1983) The synergism of GPS code and carrier measurements. In: International geodetic symposium on satellite doppler positioning, vol 1, pp 1213-1231

Hatch R (1986) Dynamic differential GPS at the centimeter level. In: Proceedings of the Fourth International Geodetic Symposium on Satellite Positioning, vol 2, pp 1287-98
Hofmann-Wellenhof B, Lichtenegger H, Collins J (2013) Global positioning system: theory and practice. Springer Science \& Business Media

ISRO (2014a) Indian regional navigation satellite system: signal in space ICD for standard positioning service, Version 1.0. ISRO Satellite Centre, June 2014

ISRO (2014b) PSLV-C22/IRNSS-1A. Available from: http://www.isro.gov.in/sites/default/files/pdf/pslvbrochures/PSLVC22.pdf, accessed 1 September 2015.

ISRO (2014c) PSLV-C24/IRNSS-1B. Available from: http://www.isro.gov.in/sites/default/files/pslv-c24brochure.pdf , published March 2014, accessed 1 September 2015

ISRO (2014d) PSLV-C26/IRNSS-1C. Available from: http://www.isro.gov.in/sites/default/files/pdf/pslvbrochures/PSLV-C26, published September 2014, accessed 1 September 2015

ISRO (2015) PSLV-C27/IRNSS-1D. Available from: http://www.isro.gov.in/sites/default/files/pdf/pslvbrochures/PSLV-C27-IRNSS-1D-BROCHURE.pdf published March 2015, accessed 1 September 2015

Kim D, Langley RB (2000) The multipath divergence problem in gps carrier-smoothed code pseudorange. In: Proceedings of 47th Annual Conference of the Canadian Aeronautics and Space, pp 161-163 
(a)

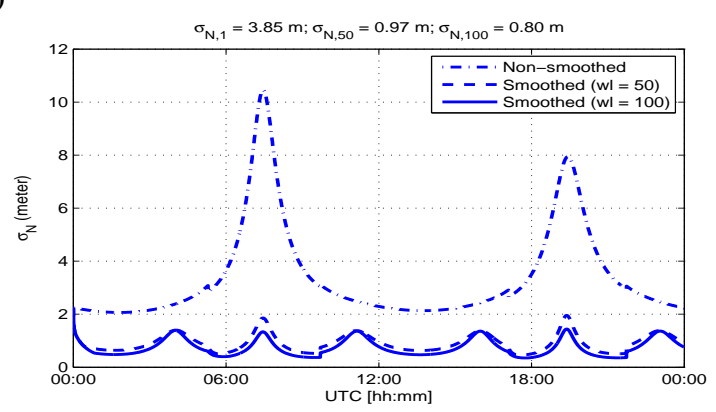

(b)

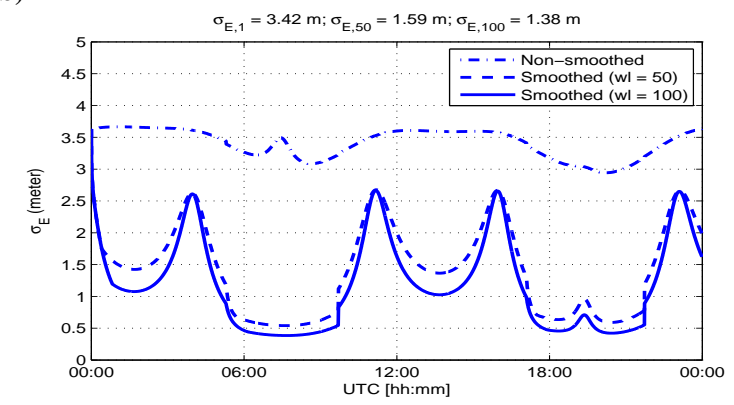

Fig. 16 Full constellation, height-constrained $\left(\sigma_{h_{r}}=5 \mathrm{~m}\right)$, horizontal SPP precision for Darwin. 'Non-smoothed' is the single epoch, pseudorange-only SPP precision; 'smoothed $(w \mathrm{l}=50)$ ' is the carrierphase smoothed pseudorange SPP precision with window length of 50 epochs; 'smoothed $(\mathrm{wl}=100)$ ' is the carrier-phase smoothed pseudorange SPP precision with window length of 100 epochs.

Kumari A, Samal K, Rajarajan D, Swami U, Babu R, Kartik A, Rathnakara SC, Ganeshan AS (2015) Precise Modeling of Solar Radiation Pressure for IRNSS Satellite. Journal of Natural Sciences Research 5(3):35-43

Montenbruck O, Steigenberger SR, Riley S (2015) IRNSS orbit determination and broadcast ephemeris assessment. In: Proceedings of the 2015 International Technical Meeting of The Institute of Navigation, Dana Point, California, January 2015, pp 185-193

Mozo Garcia A, Piriz R, Lainez Samper MD, Romay Merino MM (2010) Multisystem Real Time PrecisePoint-Positioning, today with GPS+GLONASS in the near future also with QZSS, Galileo, Compass, IRNSS. In: International Symposium on GPS/GNSS, Taipei, Taiwan, October 2010

Nadarajah N, Khodabandeh A, Teunissen PJG (2015) Assessing the IRNSS L5-signal in combination with GPS, Galileo, and QZSS L5/E5a-signals for positioning and navigation. doi:10.1007/s10291-015-0450-8. GPS Solutions pp 1-9

Odolinski R, Teunissen PJG, Odijk D (2014) First combined COMPASS/BeiDou-2 and GPS positioning results in Australia. Part I: single-receiver and relative code-only positioning. Journal of Spatial Science 59(1):3-24

Park B, Sohn K, Kee C (2008) Optimal hatch filter with an adaptive smoothing window width. Journal of Navigation
61(03):435-454

Rao VG (2013) Proposed LOS fast TTFF signal design for IRNSS. PhD thesis. University of Calgary

Rethika T, Mishra S, Nirmala S, Rathnakara SC, Ganeshan AS (2013) Single frequency ionospheric error correction using coefficients generated from regional ionospheric data for IRNSS. Indian Journal of radio \& Space Physics 42:125-130

Saastamoinen J (1972) Atmospheric correction for the troposphere and stratosphere in radio ranging satellites. The use of artificial satellites for geodesy pp 247-251

Saikiran B, Vikram V (2013) IRNSS architecture and applications. Journal of Comm \& Electron 1(3):21-27

Sarma AD, Sultana Q, Srinivas VS (2010) Augmentation of indian regional navigation satellite system to improve dilution of precision. Journal of Navigation 63(02):313321

Sekar SB, Sengupta S, Bandyopadhyay K (2012) Spectral compatibility of $\operatorname{BOC}(5,2)$ modulation with existing GNSS signals. In: Position Location and Navigation Symposium (PLANS), 2012 IEEE/ION, IEEE, pp 886-890

Teunissen PJG (1990) GPS op afstand bekeken In: een halve eeuw in de goede richting . Lustrumboek Snellius 19851990, DUM Delft pp 215-233

Teunissen PJG (1991) The GPS phase-adjusted pseudorange. In: Proceedings of the Second International Workshop on High Precision Navigation, K. Linkwitz and U. Hangleiter (eds), Dummler, Bonn, pp 115-125

Teunissen PJG (1998) A proof of Nielsen's conjecture on the relationship between dilution of precision for point positioning and for relative positioning with GPS. IEEE on Aerospace and Electronic Systems 34(2):693-695

Teunissen PJG, Amiri-Simkooei AR (2008) Least-squares variance component estimation. Journal of Geodesy 82(2):65-82

Thoelert S, Montenbruck O, Meurer M (2014) IRNSS-1A: signal and clock characterization of the Indian regional navigation system. GPS solutions 18(1):147-152

Zhao L, Li L, Zhao X (2009) An adaptive Hatch filter to minimize the effects of ionosphere and multipath for GPS single point positioning. In: Mechatronics and Automation, 2009. ICMA 2009. International Conference on, IEEE, pp 4167-4172 